\title{
On the Impact of Antenna Array Geometry on Indoor Wideband Massive MIMO Networks
}

DOI:

10.1109/TAP.2020.3008662

\section{Document Version}

Accepted author manuscript

Link to publication record in Manchester Research Explorer

\section{Citation for published version (APA):}

Temiz, M., Alsusa, E., Zhang, Y., \& Danoon, L. (2020). On the Impact of Antenna Array Geometry on Indoor Wideband Massive MIMO Networks. IEEE Transactions on Antennas and Propagation .

https://doi.org/10.1109/TAP.2020.3008662

\section{Published in:}

IEEE Transactions on Antennas and Propagation

\section{Citing this paper}

Please note that where the full-text provided on Manchester Research Explorer is the Author Accepted Manuscript or Proof version this may differ from the final Published version. If citing, it is advised that you check and use the publisher's definitive version.

\section{General rights}

Copyright and moral rights for the publications made accessible in the Research Explorer are retained by the authors and/or other copyright owners and it is a condition of accessing publications that users recognise and abide by the legal requirements associated with these rights.

\section{Takedown policy}

If you believe that this document breaches copyright please refer to the University of Manchester's Takedown Procedures [http://man.ac.uk/04Y6Bo] or contact uml.scholarlycommunications@manchester.ac.uk providing relevant details, so we can investigate your claim.

\section{OPEN ACCESS}




\title{
On the Impact of Antenna Array Geometry on Indoor Wideband Massive MIMO Networks
}

\author{
Murat Temiz, Emad Alsusa, Laith Danoon, and Yongwei Zhang \\ Department of Electrical and Electronic Engineering, \\ University of Manchester, Manchester, UK *†‡
}

\begin{abstract}
Multi-user massive multiple-input-multiple-output (massive MIMO) will play a key role in future wireless communication networks. Since spatial channel diversity is the fundamental merit of this technique, high channel correlation may significantly restrict its abilities. This study investigates the impact of channel correlation on a prototyped massive MIMO network with the objective to identify an antenna array geometry which has reduced mutual coupling and channel correlation. To this end, a highly-efficient directional wideband single antenna element was designed for the antenna arrays and the user equipments (UEs). The designed array geometry is tested in an experimental indoor wideband massive MIMO setup. Important system parameters, such as channel correlation, power delay profile, and average received power from the UEs, are studied by analyzing the measured channel data. Furthermore, system-level simulations and network capacity calculations are performed based on the measured channel data to evaluate the performance of the prototyped antenna arrays. A regular array was also fabricated and used for benchmarking comparison. Moreover, a power control algorithm is introduced for the uplink, which was shown to improve the network capacity by up to $3 \mathrm{~dB}$. The results demonstrate that the introduced antenna array outperforms the uniform antenna array in terms of mutual coupling and channel capacity.
\end{abstract}

Massive MIMO, antenna array, mutual coupling, channel correlation, power control algorithm

\section{Introduction}

Employing a large number of antennas at the BS substantially increases the capacity of the network by utilizing spatial diversity to communicate with multiple devices at the same time and frequency resources. Furthermore, it enhances energy efficiency by utilizing higher antenna gain and focusing the electromagnetic energy onto the desired UEs. The spectral and energy efficiencies of massive MIMO networks may reach 10 times, or more, than conventional networks, even with relatively simple linear receivers and precoders such as the zero-forcing (ZF) and minimum mean-square error (MMSE) receivers [2]. As these systems utilize spatial channel diversity, it is important to understand the behavior of the multiple antenna channels with different array geometries and channel conditions in an experimental network setup.

A number of studies have investigated massive MIMO channels by performing channel measurements in line-of-sight (LOS) and non-line-of-sight (NLOS) propagation environments and evaluated their performance through measured channel data $[3] 6$. The users' orthogonality and channel hardening with regard to the antenna array size were experimentally evaluated in 3 , where it was concluded that both of these features improve as the number of antennas increases. The power delay profile (PDP) and channel correlation characteristics of indoor massive MIMO networks are presented in [6], however, this study utilized virtual antenna arrays constructed by shifting a single antenna element on the $\mathrm{x}$-axis and $\mathrm{y}$-axis, thereby, did not consider the mutual coupling and the radiation pattern distortion caused by nearby elements. A similar study in 5 presented channel characteristics of outdoor massive MIMO networks based on measured data with virtual arrays. The performance of massive MIMO with uniform linear antenna (ULA) and uniform cylindrical antenna (UCA) arrays is evaluated by analyzing measured channels in [4], which revealed that the ULA array performs significantly better than the UCA array in LOS propagation as it occupies much wider space on the horizontal plane, resulting in more diverse antenna channels. The impact of mutual coupling and channel correlation on massive MIMO networks are investigated through mathematical models in 7.8 , where it was shown that both of them substantially degrade the spectral efficiency. On the other hand, some antenna elements may contribute to the system performance more than others due to fading differences among the signals received by the elements because of their locations in the array as experimentally verified in 9 .

\footnotetext{
${ }^{*}$ This work was presented in part at 2019 IEEE International Symposium on Antennas and Propagation and USNC-URSI Radio Science Meeting 1 .

$\dagger$ This study has been accepted to be published at IEEE Transactions on Antennas and Propagation, this is a preprint and is not the final version of the study which will be published by the journal.

${ }^{\ddagger}$ C 2020 IEEE. Personal use of this material is permitted. Permission from IEEE must be obtained for all other uses, in any current or future media, including reprinting/republishing this material for advertising or promotional purposes, creating new collective works, for resale or redistribution to servers or lists, or reuse of any copyrighted component of this work in other works.
} 
The effects of antenna element types with uniform array geometries on the correlation and the capacity have been investigated in 10 14. The uniform horizontal linear, the uniform vertical linear and the uniform planar antenna arrays consisting of 64 elements with half-wavelength separation are compared in [14, and it was shown that the horizontal linear array performs better than others in terms of channel separability. However, horizontal arrays are not practical since they would occupy a narrow and very long space. On the other hand, the vertical antenna array was shown to have the lowest channel orthogonality due to the fact that the channels have much richer diversity on the horizontal plane. The irregular antenna arrays are analytically examined and compared to the regular arrays in terms of mutual coupling and channel correlation in 11 which concluded that the irregular arrays may outperform regular arrays when the number of antennas is greater than a certain threshold. However, these results were not experimentally verified. Considering the antenna element radiation pattern, directional patch antenna arrays were experimentally shown to have a lower level of mutual coupling compared to dipole antenna arrays with the same element separation in [10]. It also demonstrated that directional arrays provide higher capacity than dipole antenna arrays with the $\mathrm{ZF}$ receiver [10]. In terms of array geometry, a parallelogram antenna array (PPA) was proposed to reduce the channel correlation in 12 . The performance of the PPA was evaluated using system-level simulations and experiments in $13 \mid$ where it was concluded that the PPA mitigates the overall channel correlation compared to uniform arrays. However, this study did not consider the spaces occupied by the antenna arrays, hence, the PPA geometry causes a wider antenna array size because of its parallelogram shape.

Motivated by the above, in this study, we introduce an antenna array geometry for indoor wideband massive MIMO networks and evaluate its performance in terms of mutual coupling and channel correlation through experimental channel measurements. For this purpose, a highly efficient single antenna element is designed and replicated in the arrays. To achieve a fair comparison, the total physical size and the number of antennas of the proposed array are kept the same as the uniform array benchmark. The designed single antenna element and antenna arrays were fabricated and their antenna properties were measured in the anechoic chamber and analyzed. Using these antenna arrays and single antenna elements, an indoor massive MIMO network setup, which includes UEs having various channel conditions, was constructed and channel measurements in this network were conducted. The channel data set obtained by measurements was analyzed to study channel correlation, power delay profiles, and received power variations with respect to the antenna array geometry and propagation environment in details. Using the obtained channel data, system-level simulations were performed to investigate the channel capacity of the introduced arrays and to identify any favorable channel conditions. Furthermore, a practical power control algorithm is proposed to enhance the capacity and improve the energy efficiency of the network. To the best of our knowledge, this type of comprehensive measurement, simulation and analysis based RF-system-level study which investigates the impact of array geometry on massive MIMO has not been presented in the literature before.

Although Massive MIMO BS arrays may include more than 64 antenna elements, the prototypes examined in this study were made up of 18 antenna elements. Since the size of the antenna arrays can be easily scaled up by replicating the same array, the change in mutual coupling and channel correlation in larger antenna arrays with respect to a central antenna element will be minimal due to the wavelength-separation relationship. Accordingly, relatively small array prototypes (i.e. 18 elements ) can be sufficient to investigate mutual coupling and channel correlation and their effects on the network performance. Furthermore, as the targeted application is indoor communication networks (e.g. WiFi or femtocell networks), the fabricated antenna array prototypes, consisting of $3 \times 6$ antenna elements within $226.8 \times 148.2 \mathrm{~mm}$ size, may easily fit into a typical indoor access point (AP). It is worth noting that WiFi networks have already commenced playing a significant role in sharing the cellular network load 15. By employing a massive MIMO AP in such networks, much more bandwidth may be used to provide higher data-rates to all devices.

\subsection{Contributions}

- A shifted antenna array (SRA) geometry is introduced and its performance is evaluated by performing experimental antenna and channel measurements as well as system-level massive MIMO simulations. It has been shown that the proposed antenna array geometry outperforms the uniform antenna array (URA) geometry in terms of mutual coupling level and channel correlation while occupying the same size. Furthermore, this study presents detailed analyses of the channel correlation, PDP and received power variations with respect to array geometry and channel propagation conditions in wideband indoor massive MIMO networks as well as providing analytical capacity expressions.

- A practical power control algorithm is proposed and it is shown to substantially increase the capacity of the network in addition to providing enhanced energy efficiency by reducing the output power of some UEs to their optimum levels. The proposed power control algorithm may be easily operated during the channel state information (CSI) estimation stage.

\subsection{Notation}

Throughout the paper, the following notation is used for mathematical expressions. Bold upper case letters e.g. $\mathbf{H}$ and bold lower case letters e.g. $\mathbf{h}$ denote matrices and vectors, respectively. The expected 


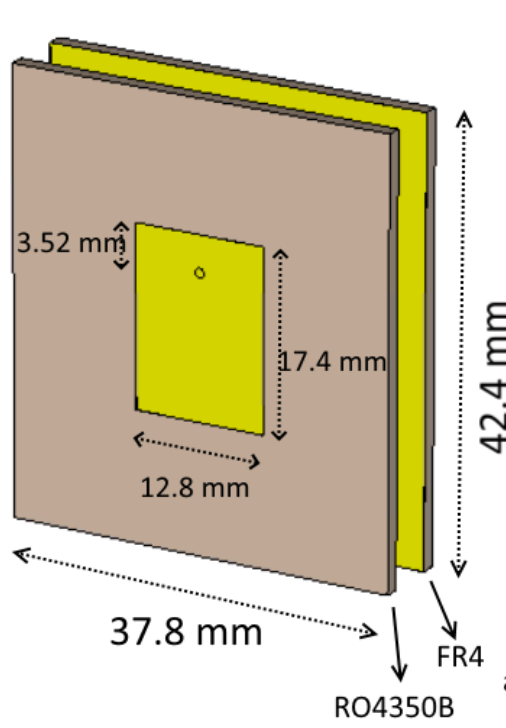

(a)

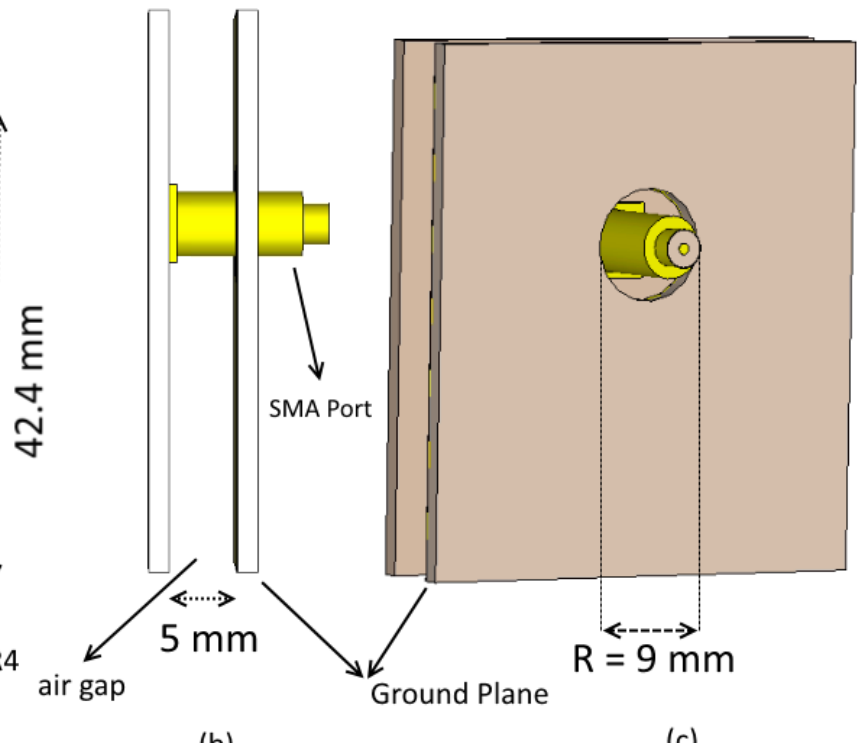

(b) (c)

Fig. 1: The designed single antenna element: (a) front, (b) side and (c) back views.

value operator is denoted by $\mathbb{E}[$.$] . Superscript [.] { }^{T},[.]^{H}$ and $[.]^{-1}$ indicate the transpose, Hermitian transpose and inverse of a matrix or vector, respectively. |.| and ||.|| denote the absolute value and $L_{2}$-norm operators, repectively.

\section{Antenna Array Design}

This section presents the designed highly-efficient single antenna and the two considered antenna arrays that consist of multiples of this element in the same physical array size.

\subsection{Single Antenna Element}

A single antenna element has been designed based on the suspended plate antenna (SPA) technique which has an air gap between the ground plane and active antenna layers. The SPA technique leads to a better radiation efficiency and increases the bandwidth, compared to microstrip antennas without causing any extra complexity in the structure [16 17]. The designed single antenna element, which is also used to compose the antenna arrays, is illustrated in Fig. 1. The substrate for the active element layer is Rogers RO4350B with relative permittivity $\varepsilon_{r}=3.48$ and dissipation factor (loss tangent) $\delta=0.0031$, which has low electrical losses at microwave frequencies. The ground plane is placed on a standard FR4 substrate. Dimensions of the active patch are $17.4 \times 12.8 \mathrm{~mm}$. The direct SMA feed port is located $3.52 \mathrm{~mm}$ below the top edge of the active element to obtain approximately $50 \Omega$ input impedance and eliminate the need for an impedance matching circuitry. The optimum air gap between the ground and active antenna layers is $5 \mathrm{~mm}$. These optimum dimensions are obtained using CST Microwave Studio, hence, the radiation efficiency of the single element reaches $92 \%$.

The bandwidth of the antenna is $700 \mathrm{MHz}$ from $4 \mathrm{GHz}$ to $4.7 \mathrm{GHz}$ according to the $\mathrm{S} 11$ measurements which also show a good agreement with the simulation results as shown in Fig. 22 . The measured radiation pattern of the antenna is shown in Fig. 2 $\mathrm{b}$. The half-power beamwidth of the single element is approximately $80^{\circ}$ on the E-plane and $100^{\circ}$ on the H-plane. The bandwidth and radiation pattern measurement results are consistent with the antenna simulations performed in the CST Microwave Studio. The measurements are carried out using a Keysight N5242A PNA-X Microwave Network Analyzer (VNA) in the anechoic chamber. The reference antenna which was used for the calibration, gain, and radiation pattern measurements was Aaronia HyperLOG 3080, a logarithmic periodic antenna which has a nearly constant gain of $5 \mathrm{dBi}$ in the range of $380 \mathrm{MHz}$ to $8 \mathrm{GHz}[18]$.

\subsection{Antenna Array}

The antenna arrays have been populated with several copies of the introduced single element. The designed URA and SRA arrays have the same physical size as shown in Fig. 3. The number of antenna elements is $3 \times 6$ and the distance between two adjacent elements is $25 \mathrm{~mm}(\sim 0.36 \lambda)$ on the horizontal axis and $32 \mathrm{~mm}(\sim 0.5 \lambda)$ on the vertical axis in the URA. In the SRA, the separation is $25 \mathrm{~mm}(\sim 0.36 \lambda)$ on the horizontal and vertical axes as shown in Fig. 3. In the SRA, some antenna elements are shifted by $16 \mathrm{~mm}$ on the vertical plane to increase the diversity of the received signals by altering the angles of incident waves to some of the array elements. The fabricated prototype arrays are presented in Fig. 4. 


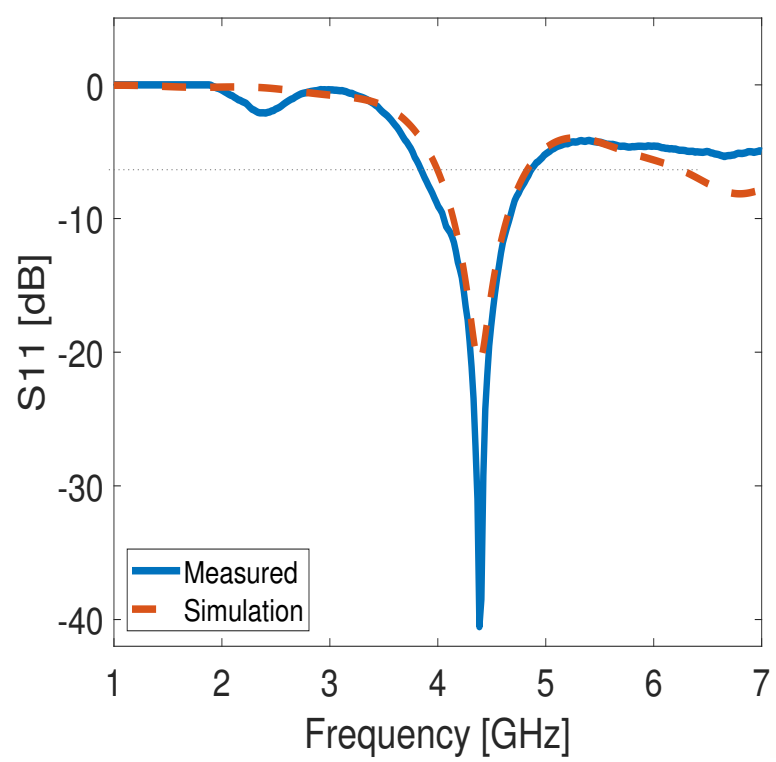

(a)

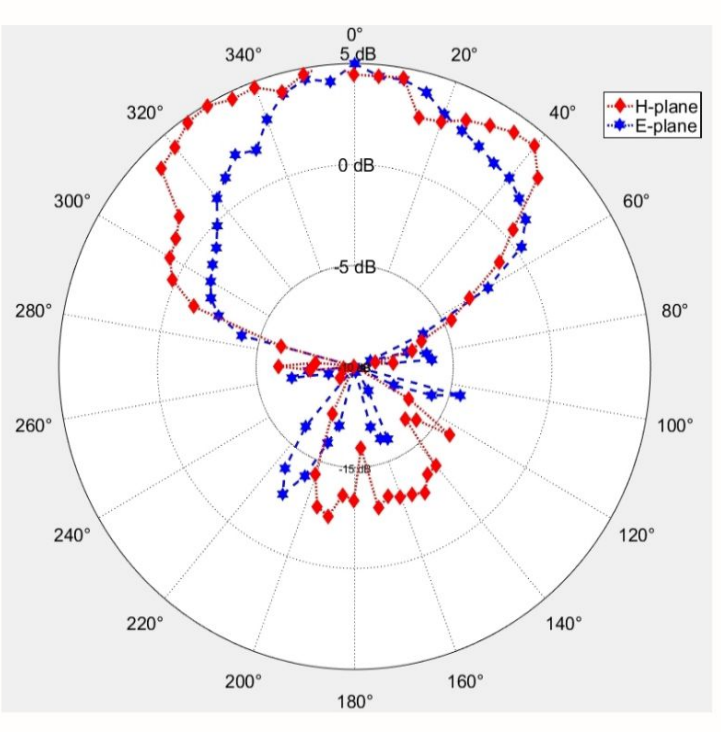

(b)

Fig. 2: (a) The measured and simulated S11 response of the single antenna and (b) measured radiation pattern of the antenna in the anechoic chamber.
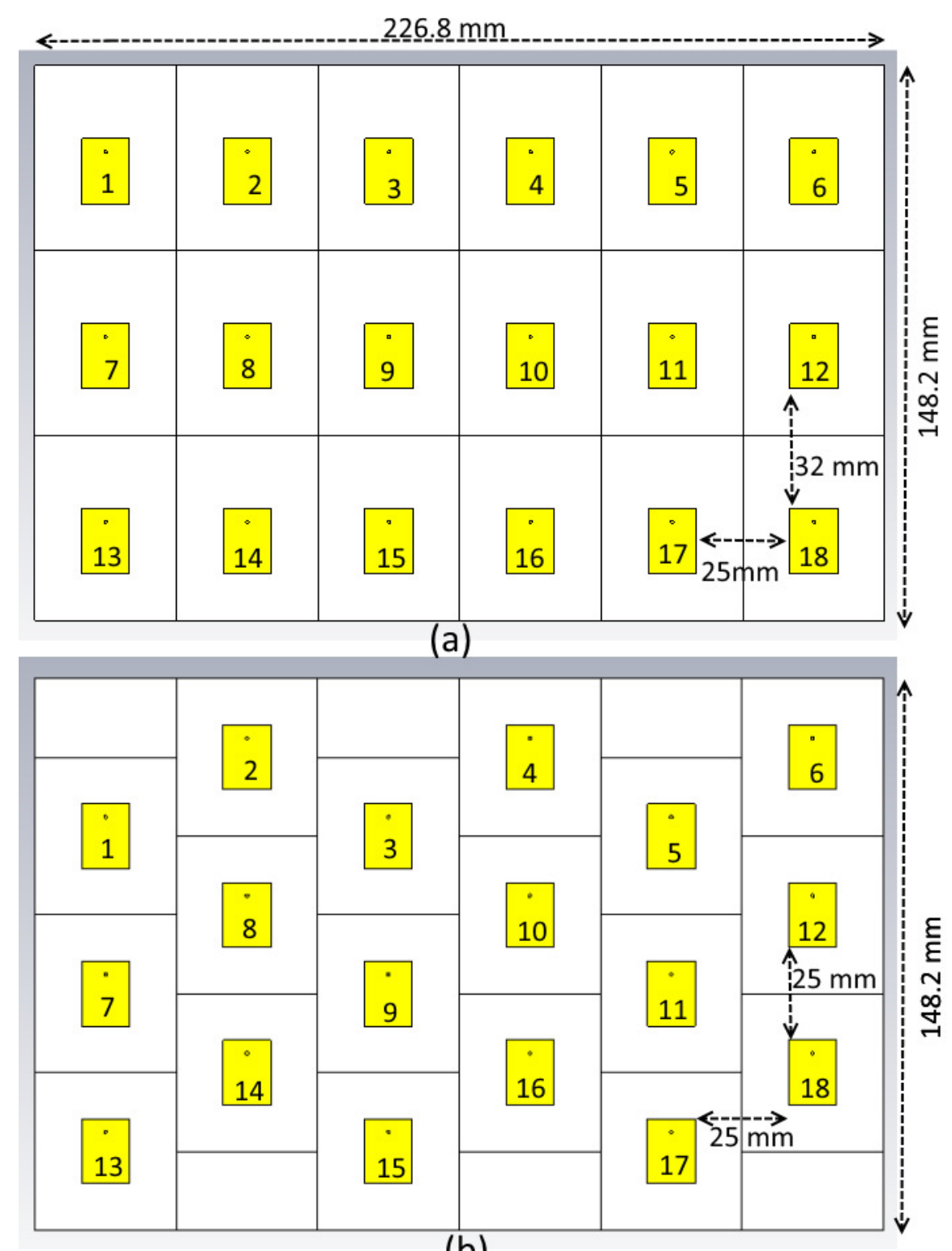

(b)

Fig. 3: (a) Uniform and (b) shifted array geometries. 


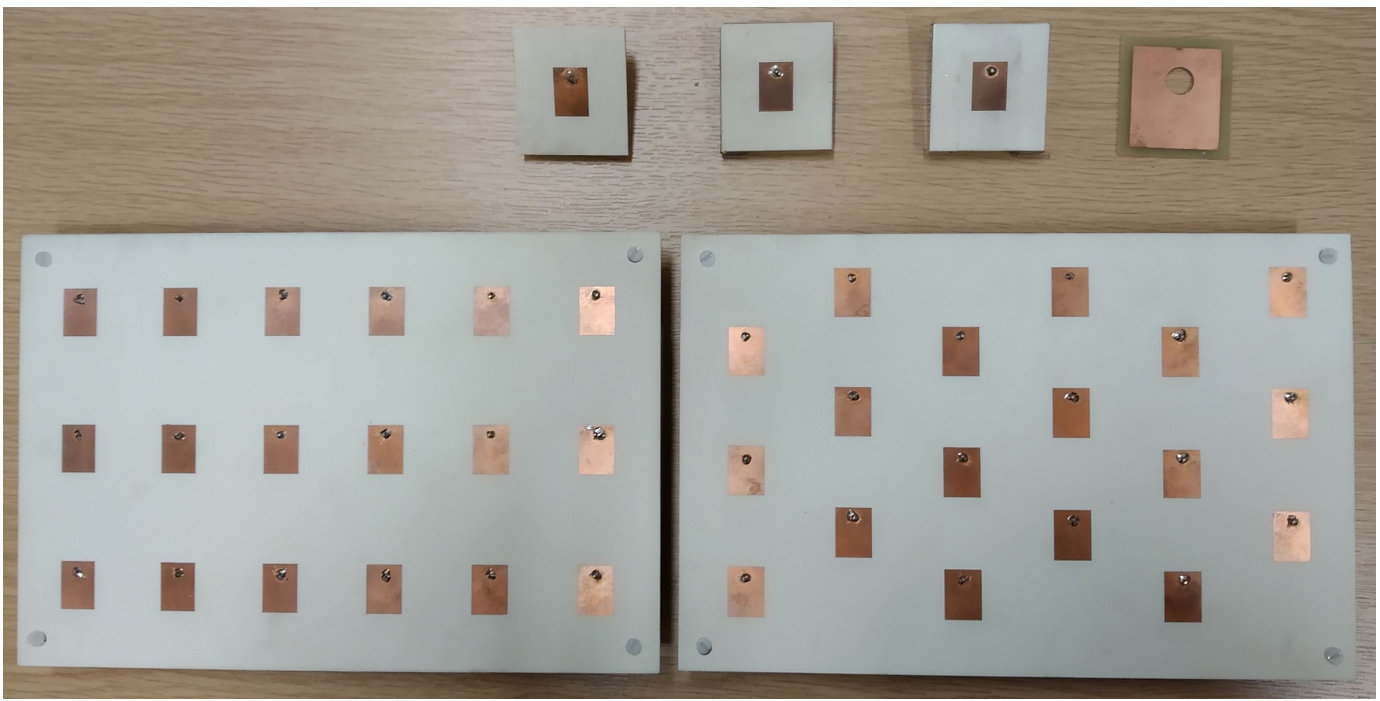

Fig. 4: Front views of the fabricated single antenna and antenna arrays. The ground plane of the antenna is presented in the top-right corner.

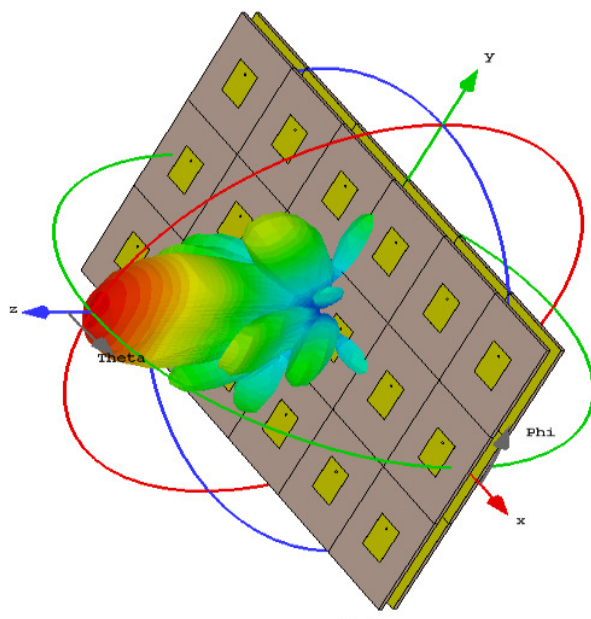

(a)

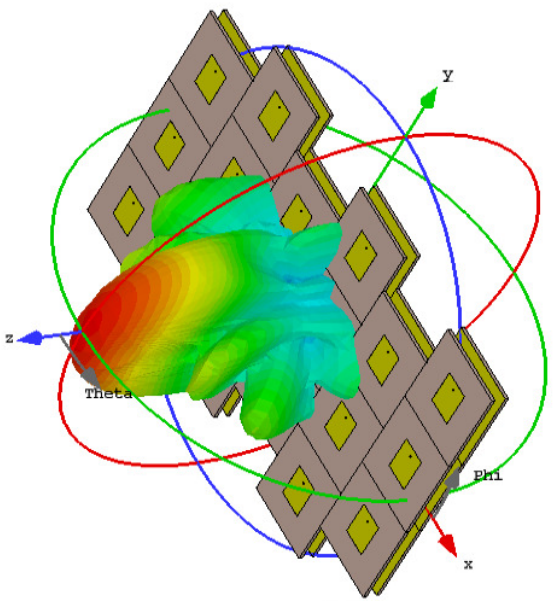

(b)

Fig. 5: Radiation patterns of the arrays: (a) URA (b) SRA.

\subsection{Radiation Pattern and Array Gains}

Assuming no strong mutual coupling among the array elements, which might distort the radiation pattern, all elements of the array will have approximately the same electric field distribution and radiation pattern. Fig. 5 shows the combined radiation patterns of the URA and the SRA at 4.4 $\mathrm{GHz}$ when all elements are excited by a unit power and the relative phases of the elements are 0 . The radiation patterns of both arrays are similar on the horizontal plane, however, the SRA has a wider radiation pattern on the vertical plane because of the shifted elements. The SRA has a lower array gain than the URA ( $18 \mathrm{~dB}$ and $16 \mathrm{~dB}$ ) as illustrated in Fig. 6 which also shows the measured and simulated gains of the single element. The main reason why the SRA has a lower gain is its wider radiation pattern on the vertical plane which is caused by shifting elements vertically which decreases the amount of power focused on the antenna boresight.

\subsection{Mutual Coupling Between The Antenna Elements}

Mutual coupling is a result of electromagnetic interaction between the antenna elements in the array [19]. It causes an electromagnetic energy transfer between them and may also give rise to correlation between the antenna channels. This effect increases as the elements are brought closer to each other. Furthermore, it tends to be stronger in the central elements since they are completely surrounded. Mutual couplings of the designed antenna arrays are simulated in CST Microwave Studio and measured using the aforementioned 4-port VNA by connecting its 4 ports to the selected 4 adjacent elements of the array while other elements are being terminated by $50 \Omega$ loads. This was repeated until all possible mutual couplings among all antenna ports are measured. The simulation and measurement results reveal that mutual coupling in the SRA array stays under $-20 \mathrm{~dB}$ while it reaches $-15 \mathrm{~dB}$ in the URA array as shown in Fig. 7. This figure presents the couplings between only the central elements of the arrays (3rd, ,4th, 9th, 10th elements) with a simulated one of the maximum mutually coupled elements $(\mathrm{S} 9,10)$. For the sake of simplicity, mutual coupling between the other elements are not included here as they have similar or lower couplings than those values presented here. The impact of mutual coupling on the capacity of MIMO systems was reviewed in [20] which concluded that high mutual coupling degrades the capacity in addition to distorting the radiation pattern of the 


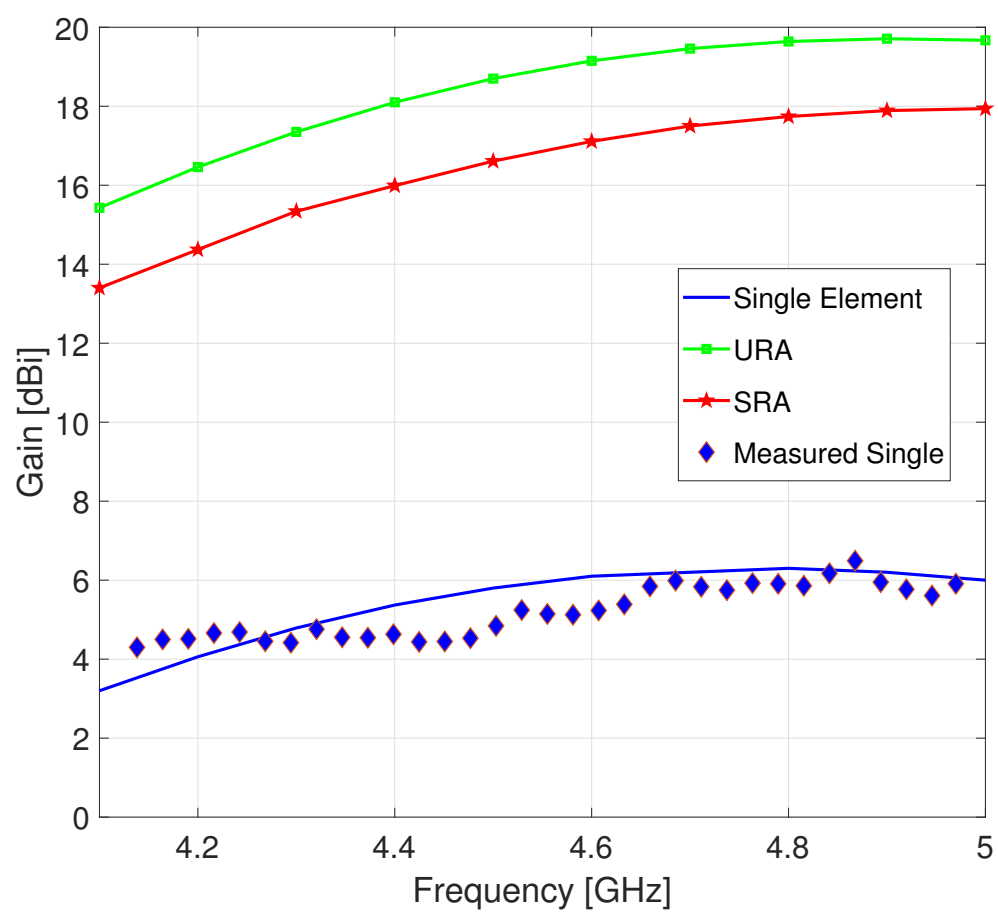

Fig. 6: The gains of the single element and the antenna arrays in simulations. Blue diamonds indicates the measured gain.

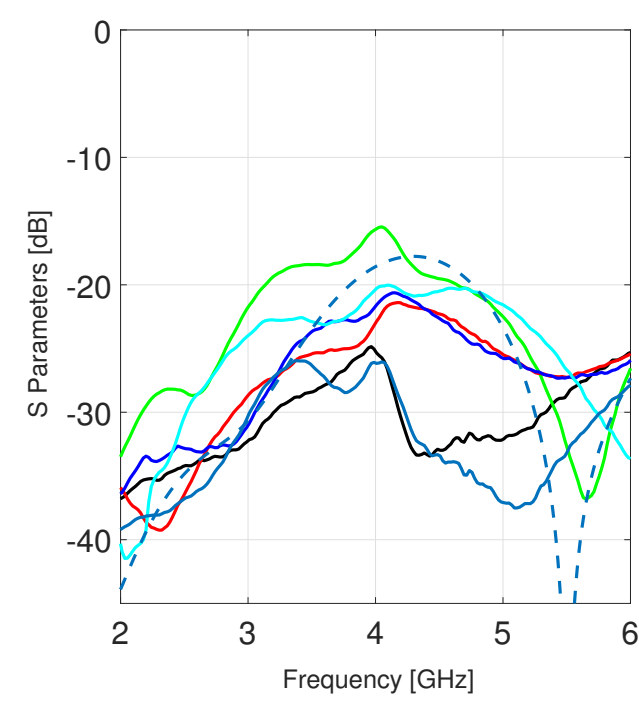

(a)

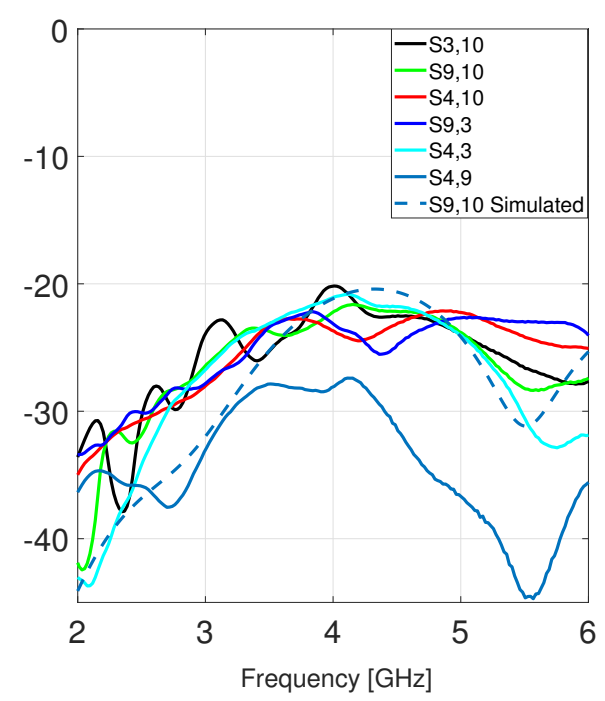

(b)

Fig. 7: Measured mutual couplings in (a) URA and (b) SRA.

array. Hence, the SRA geometry can be expected to outperform the regular array geometry in terms of channel capacity.

\section{Experimental Setup}

The measurement setup includes one BS/AP point and 8 UEs which are located in different places in an office as illustrated in Fig. 8. Each UE has only one antenna which has a directional radiation pattern as introduced in subsection 2.1. Employing directional antennas allow us to obtain strong LOS paths between the BS and the UEs when they are located facing towards each other. It must be noted here that nearly all mobile phone or $\mathrm{WiFi}$ antennas have a quasi-omnidirectional radiation pattern which is caused by the other structures around the antenna. However, as it is important to consider strong LOS paths for the performance evaluations, directional antennas have been employed in the channel measurements. The channel measurements were performed in the frequency domain using the aforementioned VNA which supports 4-port measurements up to $26.5 \mathrm{GHz}$. The bandwidth of the measurements was $700 \mathrm{MHz}$ from $4 \mathrm{GHz}$ to $4.7 \mathrm{GHz}$ and the number of samples through this frequency span was $N=7001$ points resulting in a subchannel bandwidth of $100 \mathrm{kHz}$.

The UEs are located at different positions as shown in Fig. 8 and Table 1 presents the detailed location and channel information about them. The BS array stands at $150 \mathrm{~cm}$ above the ground. UE5 and UE7 are facing towards the walls to prevent any LOS path to the BS. The rest of the UEs are facing towards the BS, hence, they might have a strong LOS path to the BS if there is no object 


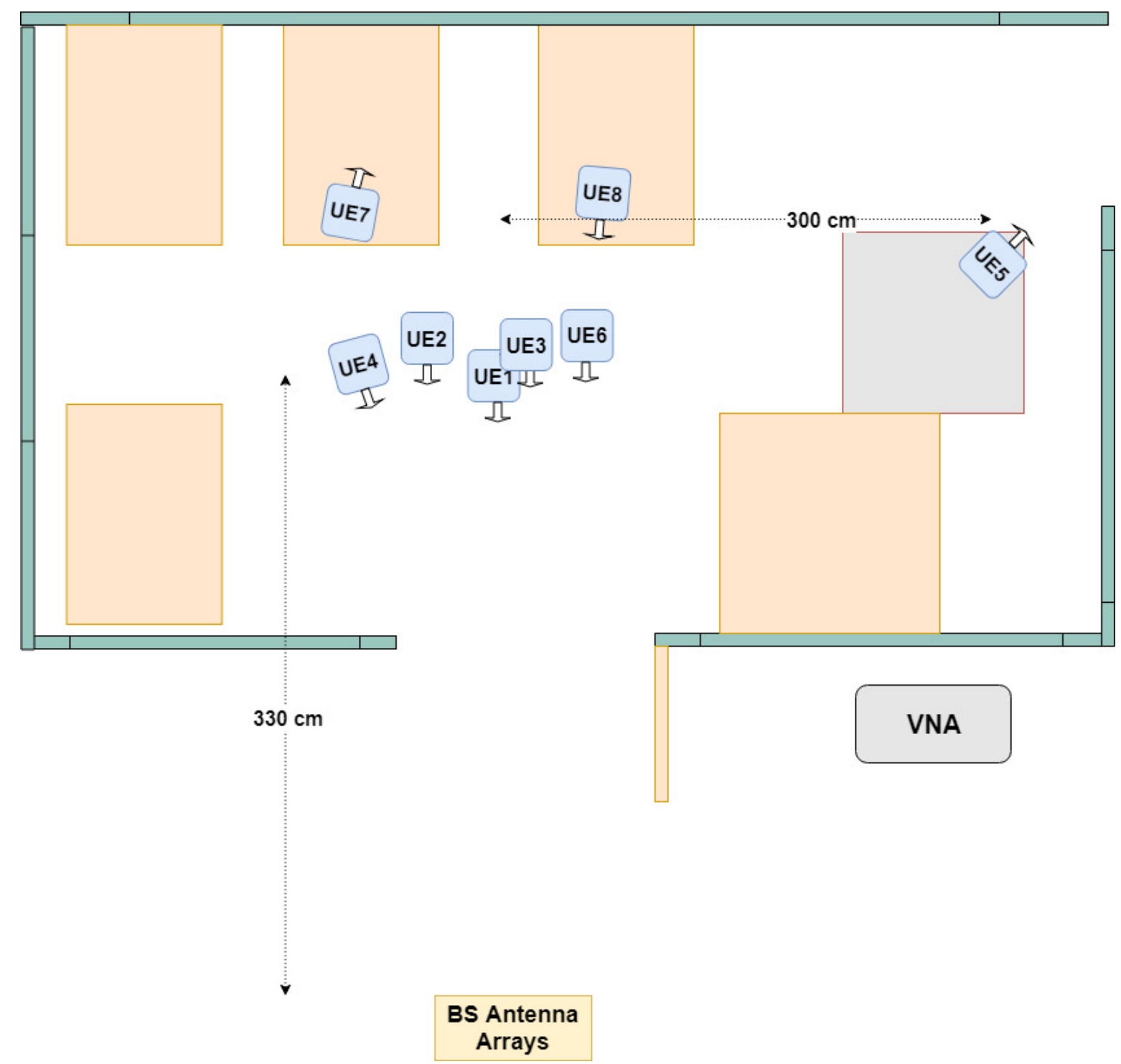

Fig. 8: Measurement setup: The BS arrays and VNA are in the first office and UEs are in the other office. Orange and the gray squares represent office desks and metal office cabinet.

Table 1: The details of the UE locations.

\begin{tabular}{|c|c|c|c|}
\hline UE & Height of the UE & Facing to & Path to the BS \\
\hline \hline UE1 & $80 \mathrm{~cm}$ & BS & not blocked \\
\hline UE2 & $80 \mathrm{~cm}$ & BS & partially blocked \\
\hline UE3 & $40 \mathrm{~cm}$ & BS & not blocked \\
\hline UE4 & $80 \mathrm{~cm}$ & BS & partially blocked \\
\hline UE5 & $140 \mathrm{~cm}$ & Wall & completely blocked \\
\hline UE6 & $80 \mathrm{~cm}$ & BS & not blocked \\
\hline UE7 & $100 \mathrm{~cm}$ & Wall & completely blocked \\
\hline UE8 & $150 \mathrm{~cm}$ & BS & not blocked \\
\hline
\end{tabular}



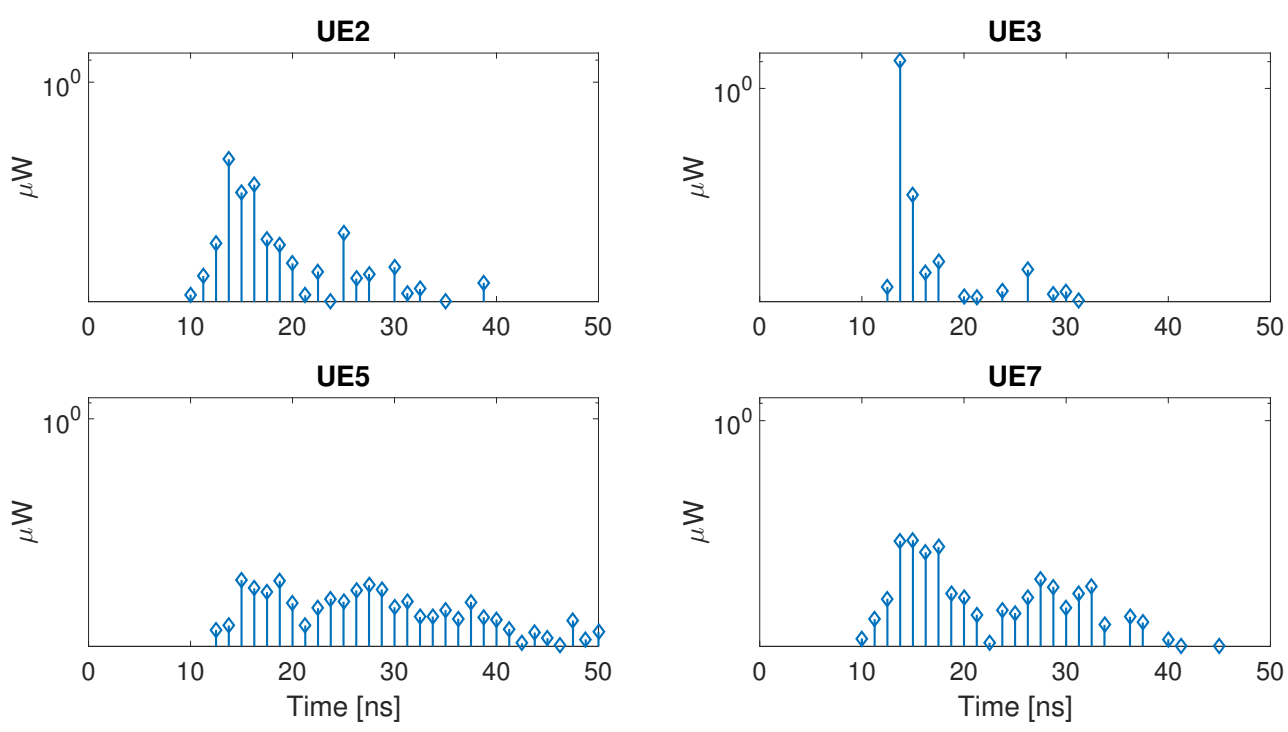

(a)
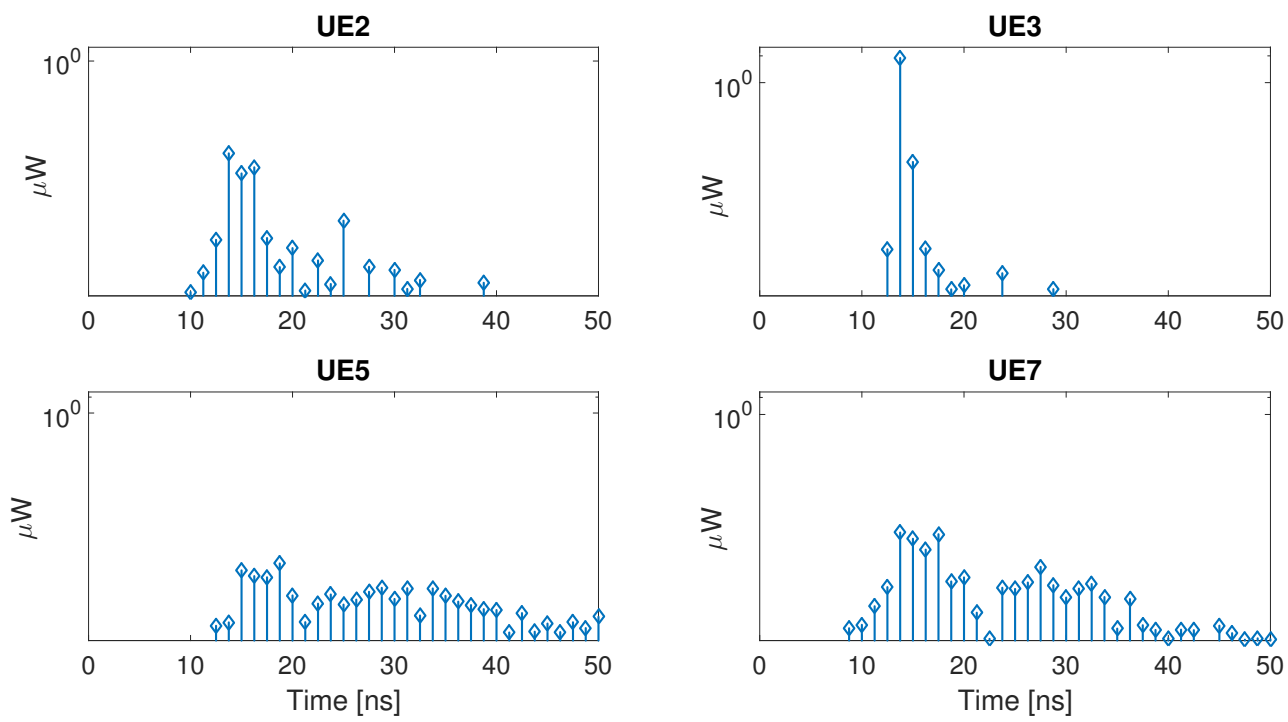

(b)

Fig. 9: Average power delay profiles of the signals received by (a) URA and (b) SRA from the UEs. .

between them. However, UE2's and UE4's radiations to the BS are partially blocked by the wall. The distance between the first UE cluster and the BS is $330 \mathrm{~cm}$, and the distance between UE5 to the first UE cluster is $300 \mathrm{~cm}$. This setup includes possible practical propagation scenarios such as closely located UEs with a strong LOS path (UE1, UE3, UE6, UE8), well separated UEs and those which do not have any LOS (UE5, UE7). Moreover, using two different array geometries allows us to investigate how correlation is affected by such array geometries, propagation environment and locations of the UEs. During channel measurements, one VNA port was connected to the UEs via a microwave switch and other three ports were connected to the arrays' ports via another switch. The switches were controlled to scan all array ports and the UEs to acquire the full channel data of the network. For each UE and antenna array configuration, the measurement data were recorded 10 times with 10 seconds intervals while the environment was constant, and the average value of them are used in order to acquire more accurate data for the correlation analysis and other presented statistics. To ensure that the channels were stationary, all measurements were performed in the evenings while no one was in the office.

Fig. 9 displays the power delay profiles (PDPs) of the selected UEs while employing the URA and SRA arrays at the BS. In this figure, the PDPs are displayed only for 4 UEs since these 4 ones have significant differences between them due to their distinct locations. The PDPs of the received signals clearly indicate the LOS and NLOS paths of the links. For instance, UE2 faces towards to the BS, however, its paths to the BS are partially blocked, therefore, it has a weaker LOS path and more NLOS paths. UE5 and UE7 do not have any LOS paths due to their directions, thus, only reflected paths from the objects are observed for these UEs with a much longer delay spreads compared to UE3.

\subsection{Channel Correlation}

Channel correlation worsens CSI errors during channel estimation and makes symbol detection more difficult by increasing the interference between the UEs [21]. In TDD systems, since the channels are reciprocal during each coherence time, the correlation is also anticipated to have reciprocity. Therefore, we analyze the channel correlation only during the uplink. Channel correlation can be classified into two categories; intra-UE correlation and inter-UEs correlation. Intra-UE correlation occurs between the channels of the same UE, therefore, it can be constructively utilized in some cases such as for the 
detection of the UE locations. Inter-UEs correlation occurs between the channels of different UEs and degrades their performance. In this study, both types of correlation are investigated. The channel correlation coefficient $(\phi)$ indicates the similarity between two channels, which is a complex number since the channel coefficients have real and imaginary parts. The magnitude of the correlation $|\phi|$ is between 0 (for entirely uncorrelated channels) and 1 (for fully correlated channels). The correlation coefficient $\phi\left(\mathbf{h}_{i}, \mathbf{h}_{j}\right)$ between the $i$ th and the $j$ th channels is given by 22

$$
\phi\left(\mathbf{h}_{i}, \mathbf{h}_{j}\right)=\frac{\mathbb{E}\left[\mathbf{h}_{i}^{H} \mathbf{h}_{j}\right]}{\sqrt{\mathbb{E}\left[\left\|\mathbf{h}_{i}\right\|^{2}\right] \mathbb{E}\left[\left\|\mathbf{h}_{j}\right\|^{2}\right]}},
$$

where $\mathbf{h}_{i} \in \mathbb{C}^{N \times 1}$ and $\mathbf{h}_{j} \in \mathbb{C}^{N \times 1}$ are the channel vectors of the $i$ th and the $j$ th channels which consist of $N=7001$ complex channel gains between $4 \mathrm{GHz}$ and $4.7 \mathrm{GHz}$. Since channel measurements are repeated 10 times, the expectation of these values are taken to find the average correlation of each channel pair. These two channel vectors are extracted from the measured full channel data by slicing it along the frequency dimension $N$, i.e. $\left\{\mathbf{h}_{i}^{T} \in \mathbb{C}^{1 \times N}, \mathbf{h}_{j}^{T} \in \mathbb{C}^{1 \times N}\right\} \in \mathbf{H}^{M \times K \times N}$. It is worth noting that the total number of channels is $M \times K=144$ which turns out $144 \times 144$ correlation pairs for 18 BS antennas and 8 UEs.

Fig. 10 presents the channel correlation among all measured channels with the URA and the SRA. Taking into account the locations of the UEs given in Fig. 8 and the PDPs of the received signals given in Fig. 9, it is clear that the UEs having strong LOS path have a higher correlation between their own channels. Furthermore, when the UEs are close to each other in LOS, the inter-UEs correlation may reach up to 0.8. For example, UE1, UE3, and UE6 are closer to each other and have very strong LOS paths, hence, the inter-UE correlation is significantly higher. UE5 and UE7 do not have any dominant LOS paths, hence, the correlation is significantly lower even between their own channels. The channel correlation of UE8 exhibits very interesting behavior, it has a very high correlation among its channels, however, it seems to be uncorrelated with the other UEs. The main reason for this outcome is its height which is significantly higher than the other UEs and at the same height as the BS. Therefore, its signals arrive at the BS at different angles than the rest. The trend of the correlation is very similar in both arrays, which shows that the measurements were reliable. A more detailed analysis of the correlation is given in Fig. 11 which illustrates the average intra-UE correlations and average inter-UEs correlations between the most correlated UEs (UE1, UE3 and UE6) with the URA and SRA geometries. The maximum correlation was observed among UE3's channels as $|\phi|=0.8935$ and the lowest correlation was observed among UE5's channels as $|\phi|=0.0468$. It reveals that the SRA geometry reduces the correlation in LOS, especially for the UEs those are highly correlated. Therefore, using the SRA geometry in the same physical size would be beneficial to reducing channel correlation and alleviating mutual coupling.

\subsection{Average Received Power}

The maximum transmit power of the UEs is generally $20 \mathrm{dBm}$ in $2.4 \mathrm{GHz}$ and $23 \mathrm{dBm}$ in $5 \mathrm{GHz}$ indoor networks for $40 \mathrm{MHz}$ bandwidth [23. In this study, the maximum transmit power of the UEs is assumed to be $30 \mathrm{dBm}$ since it is a wideband system which experiences a much higher level of thermal noise. The average received power by each antenna element of the BS from the $k$ th UE is calculated from the measured channel data by $p_{r, k}=p_{u, k} / M \sum_{n=1}^{N} \sum_{m=1}^{M} \mathbb{E}\left[\left|h_{m, k, n}\right|^{2}\right]$, where $p_{u, k}$ denotes the transmit power of the $k$ th UE. $h_{m, k, n}$ denotes the complex channel coefficient of the measured channel between the $k$ th UE and the $m$ th element of the antenna array in the $n$th subchannel. Some variations between the average powers received by two array geometries are observed due to the different locations of the elements in the arrays. Fig. 12 presents the average received powers by the BS with each array geometry from each UE. Having directional antennas, the received powers from the UEs which have LOS link reached $-58 \mathrm{dBm}$. However, the average received power from UE5 was $-72 \mathrm{dBm}$ as it does not have any LOS link. There is a significant difference (around $14 \mathrm{dBm}$ ) between received powers of UE5 and UE3 which have the lowest and the highest received powers by the BS, respectively. Therefore, a power control algorithm is introduced to adjust the power of the UEs to minimize the interference and balance the received power differences among the UEs. In wideband systems, the noise induced by electronic components is substantially higher than narrowband systems. A state-of-art ultra-wideband (500 MHz bandwidth) receiver, which consists of low noise amplifiers, RF chain and $\mathrm{ADCs}$, is reported to have about $7 \mathrm{~dB}$ noise figure 24]. It is widely accepted that the noise power spectral density is $-174 \mathrm{dBm} / \mathrm{Hz}$ at $290 \mathrm{~K}\left(16.85^{\circ} \mathrm{C}\right)$ temperature for $50 \Omega$ communication systems. For $500 \mathrm{MHz}$ bandwidth, the total thermal noise power is estimated as $-87 \mathrm{dBm}$. Therefore, the total noise power including thermal noise and receiver noise figure is $-80 \mathrm{dBm}$. Taking into account the received powers by each BS antenna array as given in Fig. 12, this system can effectively operate at $500 \mathrm{MHz}$ bandwidth to communicate all UEs within the scenario given in Fig. 8

\section{Capacity of Measured Channels}

This section presents the capacity analyses of the measured channels for the URA and the SRA. The capacity of the massive MIMO network with both arrays is also computed and compared to the performance of the independent and identically random (i.d.d.) channel. Furthermore, the capacity enhancement provided by the power control algorithm is presented here. 


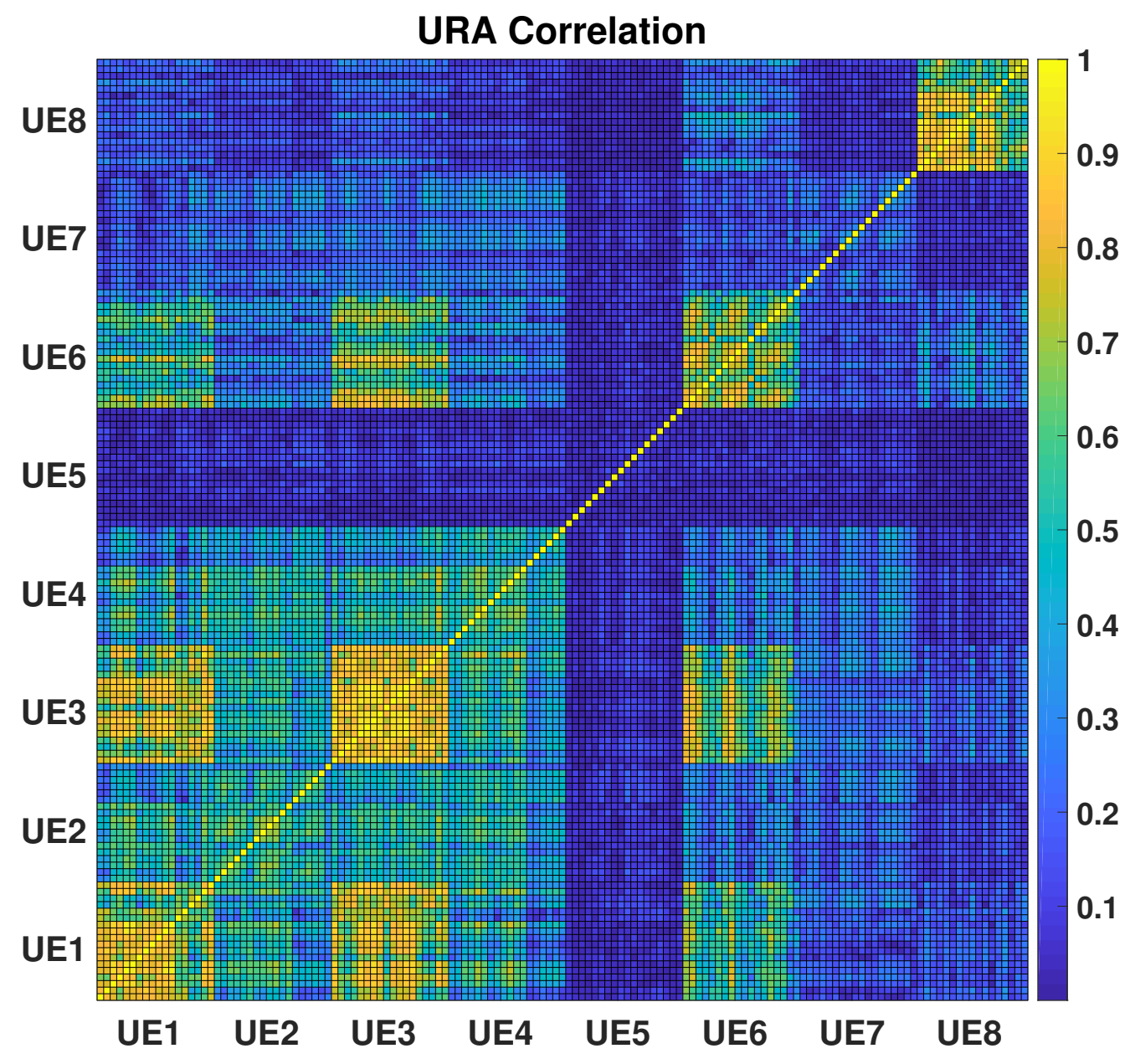

(a)

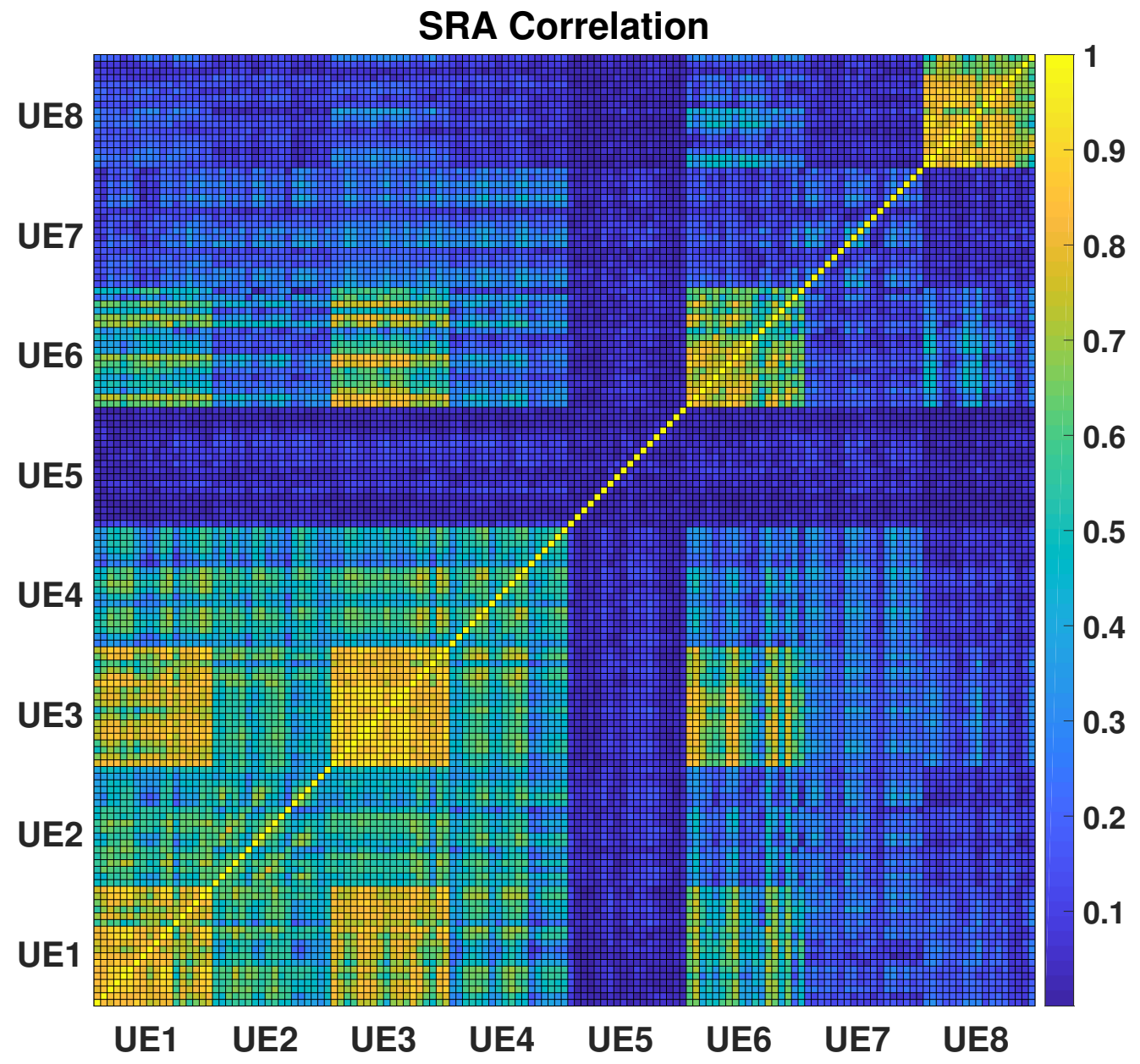

(b)

Fig. 10: Measured channel correlations $(|\phi|)$ among all UE channels with (a) URA and (b) SRA. 


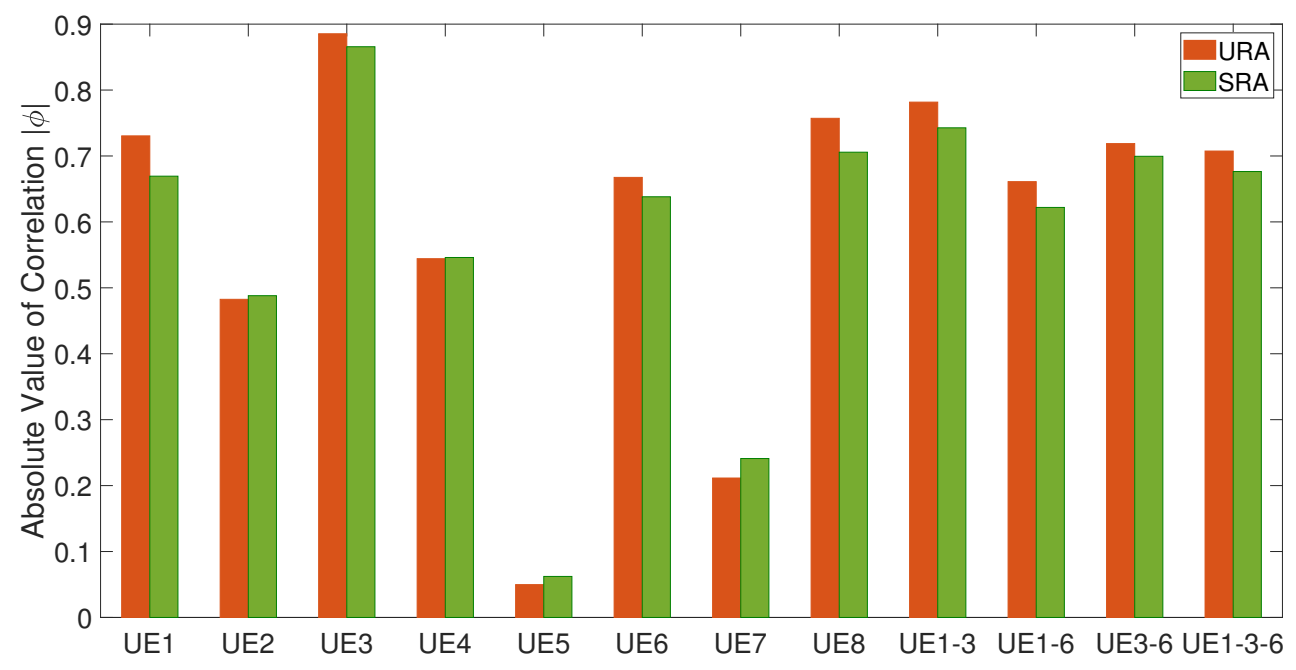

Fig. 11: Average intra-UE correlations of each UE and inter-UEs correlations among UE1, UE3 and UE6.

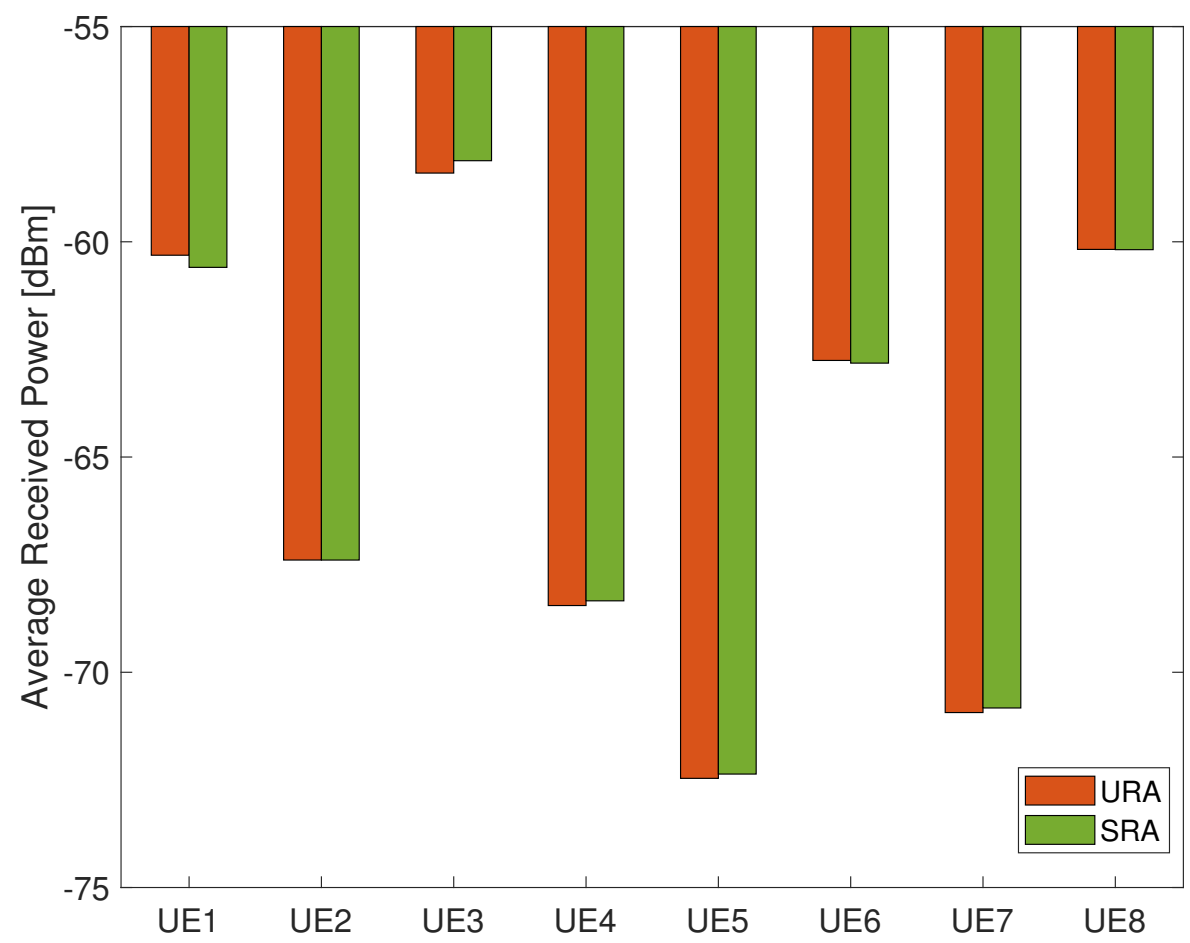

Fig. 12: Average received powers by the arrays from UEs. 


\subsection{Channel Model}

A number of channel models have been developed for MIMO and massive MIMO systems, a detailed comparison of these models is presented in [25]. In this study, we use a channel model based on the ray tracing and stochastic channel parameters. Let $G_{m, k, n, i}$ be the antenna gain along the $i$ th path between the $m$ th BS antenna and the $k$ th UE in the $n$th subchannel, which consists of the transmitter and receiver antenna gains along the $i$ th path as $G_{m, k, n, i}=G_{r, m, k, n, i} G_{t, m, k, n, i}$. This channel model considers the location of the UEs, reflection of the rays and the gain of the antennas. The time domain baseband channel between the $m$ th antenna and $k$ th UE in the $n$th subchannel is given by $h_{m, k, n}(t)=h_{L O S, m, k, n}(t)+h_{N L O S, m, k, n}(t)$, where

$$
\begin{gathered}
h_{L O S, m, k, n}(t)=\frac{\lambda}{4 \pi} \sqrt{\frac{\Lambda}{\Lambda+1}} \frac{\sqrt{G_{m, k, n}}}{d_{m, k, n}} \exp \left(-\frac{j 2 \pi d_{m, k, n}}{\lambda}\right), \\
h_{N L O S, m, k, n}(t)=\frac{\lambda}{4 \pi} \sqrt{\frac{1}{(\Lambda+1)(L+1)}} \sum_{i=1}^{L} \delta\left(t-\tau_{m, k, n, i}\right) \times \frac{\sqrt{R G_{m, k, n, i}}}{d_{m, k, n, i}} \exp \left(-\frac{j 2 \pi d_{m, k, n, i}}{\lambda}\right),
\end{gathered}
$$

where $\lambda$ denotes the wavelength of the carrier frequency and $\tau_{m, k, n, i}=\left(d_{m, k, n}-d_{m, k, n, i}\right) / c$ denotes the delay of the $i$ th reflected path due to the reflections, and it is indicated by the delta function i.e. $\delta\left(t-\tau_{m, k, n, i}\right) \cdot \sqrt{\frac{\Lambda}{\Lambda+1}}$ denotes the amplitude of the LOS path and $\sqrt{\frac{1}{(\Lambda+1)(L+1)}}$ denotes the amplitude of each NLOS path with regard to the Rician K-factor $\Lambda$ and the number of total paths $L$. The power loss along the path caused by reflections is denoted by $R$, which is the power ratio between the incident and reflected waves from an object. This value is dependent on the material, thickness, size of the object and the angle of the incident waves $\sqrt{26}$. The frequency response of the channel is calculated by the Fourier transform as $h_{m, k, n}=\int_{-\infty}^{\infty} h_{m, k, n}(t) e^{-j 2 \pi f t} d t$. Accordingly, the frequency domain channel matrix $\mathbf{H}_{n}$ in the $n$th subchannel is given by

$$
\mathbf{H}_{n}=\left[\begin{array}{ccc}
h_{1,1, N} & \cdots & h_{1, K, N} \\
\vdots & \ddots & \vdots \\
h_{M, 1, N} & \cdots & h_{M, K, N}
\end{array}\right] .
$$

\subsection{Channel Capacity Analysis}

The capacity of the prototyped network has been investigated using the measured channel data. For the channel capacity analysis, we consider a single-cell multi-user massive MIMO network which consists of one BS and 8 UEs as in the measurement setup. TDD network operation mode is selected since it enables the utilization of the channel reciprocity by using the same channel for the uplink and downlink, alternately. The uplink is considered for the capacity analyses as uplink and downlink would have similar channel capacities due to the channel reciprocity. The frequency-domain baseband received signal by the BS in each OFDM subcarrier is given by

$$
\mathbf{y}=\mathbf{H} \sqrt{p_{u}} \mathbf{x}+\mathbf{n},
$$

where, $\mathbf{H} \in \mathbb{C}^{M \times K}, \mathbf{x}=\left[\mathrm{x}_{1}, \mathrm{x}_{2}, \cdots, \mathrm{x}_{\mathrm{K}}\right]^{T} \in \mathbb{C}^{K \times 1}$, and $\mathbf{y}=\left[y_{1}, y_{2}, \cdots, y_{M}\right]^{T} \in \mathbb{C}^{M \times 1}$ denote the channel matrix among the BS and the UEs, the transmitted QAM symbol vector by $K$ UEs, and the received symbol vector by $M$ BS antennas. The channel gain between the $k$ th UE and the $m$ th antenna, $h_{m k}$, is assumed to be constant during the coherence time. $p_{u}$ denotes the average transmit power of each $\mathrm{UE}$ and $\mathbf{n}=\left[n_{1}, n_{2}, \ldots, n_{M}\right]^{T} \in \mathbb{C}^{M \times 1}$ denotes the AWGN noise vector consisting of $n_{m} \sim \mathcal{C N}\left(0, \sigma_{n}^{2}\right)$ with noise variance $\sigma_{n}^{2}$. Assuming that the symbols have a unit amplitude as $\mathbb{E}\left[\left|x_{k}\right|\right]=1$, the average SNR for the received signal from the $k$ th UE can be given by $\rho_{k}=p_{u, k} \mathbb{E}\left[\frac{\left\|\mathbf{h}_{k}\right\|^{2}}{\|\mathbf{n}\|^{2}}\right]$, where $\mathbf{h}_{k} \in \mathbb{C}^{M \times 1}$ denotes the channel vector of the $k$ th UE. Let $\hat{\mathbf{H}}$ denote the estimated CSI which consists of estimation errors due to various reasons such as AWGN noise, discrepancies between the transmitter and receiver RF chains, interference induced by other devices and channel correlation 21. Especially at low SNR values, it is more challenging to estimate the channel perfectly as the random noise dominates the received signal. The estimated CSI can be given by $\hat{\mathbf{H}}=\mathbf{H}+\mathbf{E}$, where the error matrix $\mathbf{E}$ consists of the deviations from the real channel. When CSI is perfectly estimated using pilot symbols (perfect CSI case), $\mathbf{E}=0$. In this study, the BS is assumed to employ the minimum mean square error (MMSE) estimator since it performs well in massive MIMO systems. The MMSE estimator is given in [2, 27]. When the channels are uncorrelated, this estimator can estimate the channel more precisely. However, the channel data acquired by measurements includes correlations and significant power variations among the UEs. Consequently, the channel estimation accuracy is used as a benchmark to compare the antenna arrays with the favorable (uncorrelated) channels. To this end, the mean squared error (MSE) of the estimated channel matrix is computed by

$$
M S E=\frac{1}{K} \sum_{k=1}^{K} \frac{\mathbb{E}\left[\left\|\hat{\mathbf{h}}_{k}-\mathbf{h}_{k}\right\|^{2}\right]}{\mathbb{E}\left[\left\|\mathbf{h}_{k}\right\|^{2}\right]} .
$$




$$
R_{k}=\sum_{n=1}^{N} B \log _{2}\left(1+\frac{p_{u s} \mathbb{E}\left[\left|\mathbf{w}_{k, n}^{H} \hat{\mathbf{h}}_{k, n} x_{k, n}\right|^{2}\right]}{p_{u s} \mathbb{E}\left[\left|\sum_{i=1, i \neq k}^{K} \mathbf{w}_{k, n}^{H} \hat{\mathbf{h}}_{i, n} x_{i, n}\right|^{2}\right]+p_{u s} \mathbb{E}\left[\left|\sum_{i=1}^{K} \mathbf{w}_{k, n}^{H} \varepsilon_{i, n} x_{i, n}\right|^{2}\right]+\mathbb{E}\left[\left\|\mathbf{w}_{k, n}^{H}\right\|^{2} \sigma_{n}^{2}\right]}\right)
$$

It is assumed that the BS performs data detection to acquire the message vectors transmitted by the UEs by utilizing the estimated channel matrix $\hat{\mathbf{H}}$ and employing the ZF receiver which is given by $\mathbf{W}=\hat{\mathbf{H}}\left(\hat{\mathbf{H}}^{H} \hat{\mathbf{H}}\right)^{-1} \in \mathbb{C}^{M \times K}$. Consequently, the received message vector $\hat{\mathbf{x}}$ from all UEs is given by

$$
\hat{\mathbf{x}}=\mathbf{W}^{H} \mathbf{y}=\mathbf{W}^{H}\left(\mathbf{H} \sqrt{p_{u}} \mathbf{x}+\mathbf{n}\right) .
$$

The received signal by the BS from the $k$ th UE can be explicitly written as

$$
\hat{x}_{k}=\sqrt{p_{u}} \mathbf{w}_{k}^{H} \hat{\mathbf{h}}_{k} x_{k}+\sqrt{p_{u}} \sum_{i=1, i \neq k}^{K} \mathbf{w}_{k}^{H} \hat{\mathbf{h}}_{i} x_{i}+\sqrt{p_{u}} \sum_{i=1}^{K} \mathbf{w}_{k}^{H} \boldsymbol{\varepsilon}_{i} x_{i}+\mathbf{w}_{k}^{H} \mathbf{n},
$$

where $\boldsymbol{\varepsilon}_{i} \in \mathbb{C}^{M \times 1}$ is the CSI error vector for $i$ th UE, hence $\hat{\mathbf{h}}_{i}=\mathbf{h}_{i}+\boldsymbol{\varepsilon}_{i}$ and $\mathbf{w}_{k} \in \mathbb{C}^{M \times 1}$ denotes the $k$ th column vector of the $\mathbf{W}$, corresponding to the ZF receiver for the $k$ th UE. The received signal-to-interference-noise ratio (SINR) $\gamma_{k}$ for $k$ th UE is then given by

$$
\gamma_{k}=\frac{p_{u} \mathbb{E}\left[\left|\mathbf{w}_{k}^{H} \hat{\mathbf{h}}_{k} x_{k}\right|^{2}\right]}{p_{n k}},
$$

where $p_{n k}$ denotes power of the noise and interference including CSI errors for the $k$ th UE, is given by

$$
p_{n k}=p_{u} \mathbb{E}\left[\left|\sum_{i=1, i \neq k}^{K} \mathbf{w}_{k}^{H} \hat{\mathbf{h}}_{i} x_{i}\right|^{2}\right]+p_{u} \mathbb{E}\left[\left|\sum_{i=1}^{K} \mathbf{w}_{k}^{H} \varepsilon_{i} x_{i}\right|^{2}\right]+\mathbb{E}\left[\left\|\mathbf{w}_{k}^{H} \mathbf{n}\right\|^{2}\right] .
$$

For the measured channel data with CSI mismatch, the achievable capacity is computed by (11) where average transmit power per subchannel is $p_{u s}=p_{u} / N$ and subscript $n$ indicates the index of the OFDM subcarriers i.e. $n=1,2, \ldots N, B$ denotes the bandwidth of each OFDM subcarrier i.e. $B=100 \mathrm{kHz}$, and the total bandwidth is $500 \mathrm{MHz}$ resulting in $N=5000$ subchannels.

\subsection{Power Control Algorithm}

The channel measurements have shown that significant variations in the received powers from the UEs can be observed since each channel has a substantially different gain as UEs are randomly scattered in the environment. For instance, having a strong LOS path increases the channel gain in addition to the channel correlation. For capacity bound analysis, it is usually assumed that the received powers from all UEs are distributed with the same mean and variance under favorable channel conditions, unlike the measurement results demonstrated. Therefore, a power control algorithm which computes the received powers from each UE then directs the UEs to reduce or increase their powers with respect to their channel gains will be beneficial. Conventional water-filling power control (WFP), which is based on convex optimization, endeavors to maximize the sum capacity of the network under a fixed sum-power constraint [28]. The WFP is widely used for downlink and can be implemented iteratively since it is an optimization problem which may cause a high computational complexity. Furthermore, it maximizes the sum-capacity of the network without considering the capacity of each UE. Hence, we introduce a practical uplink power control (PC) algorithm which equilibrates average received powers of UEs to ensure that all UEs can achieve similar capacities without causing any significant interference on the other UEs. This PC algorithm is implemented in the uplink channel estimation stage to adjust the UE powers in each TDD frame instantaneously. There are two main advantages of having power control in uplink massive MIMO networks. Firstly, it reduces the interference caused by the UEs which are closer to the BS or have strong LOS paths by controlling their output powers. Secondly, it decreases the power consumption of the UEs while providing the same or better SINR for the whole network. This enhances the energy efficiency of the network as well as the spectral efficiency.

The PC algorithm presented in Algorithm 1 firstly computes the received powers from the UEs during CSI estimation. Then, it computes the channel gain of each UE and aims at maximizing the received powers from the UEs which have the lowest channel gains. $T_{S N R}$ is defined as the SNR of the UE with the lowest channel gain, $\hat{\mathbf{h}}_{k_{m i n}, n}$, at its maximum power output. Afterwards, it commands other UEs to decrease their transmit powers to the specifically calculated power levels for each UE to equilibrate all received average powers from the UEs. The output power of each UE is limited by $p_{\max }=30 \mathrm{dBm}$ i.e. $0<p_{u, k} \leq p_{\max }$. This kind of power control algorithm can be easily implemented 


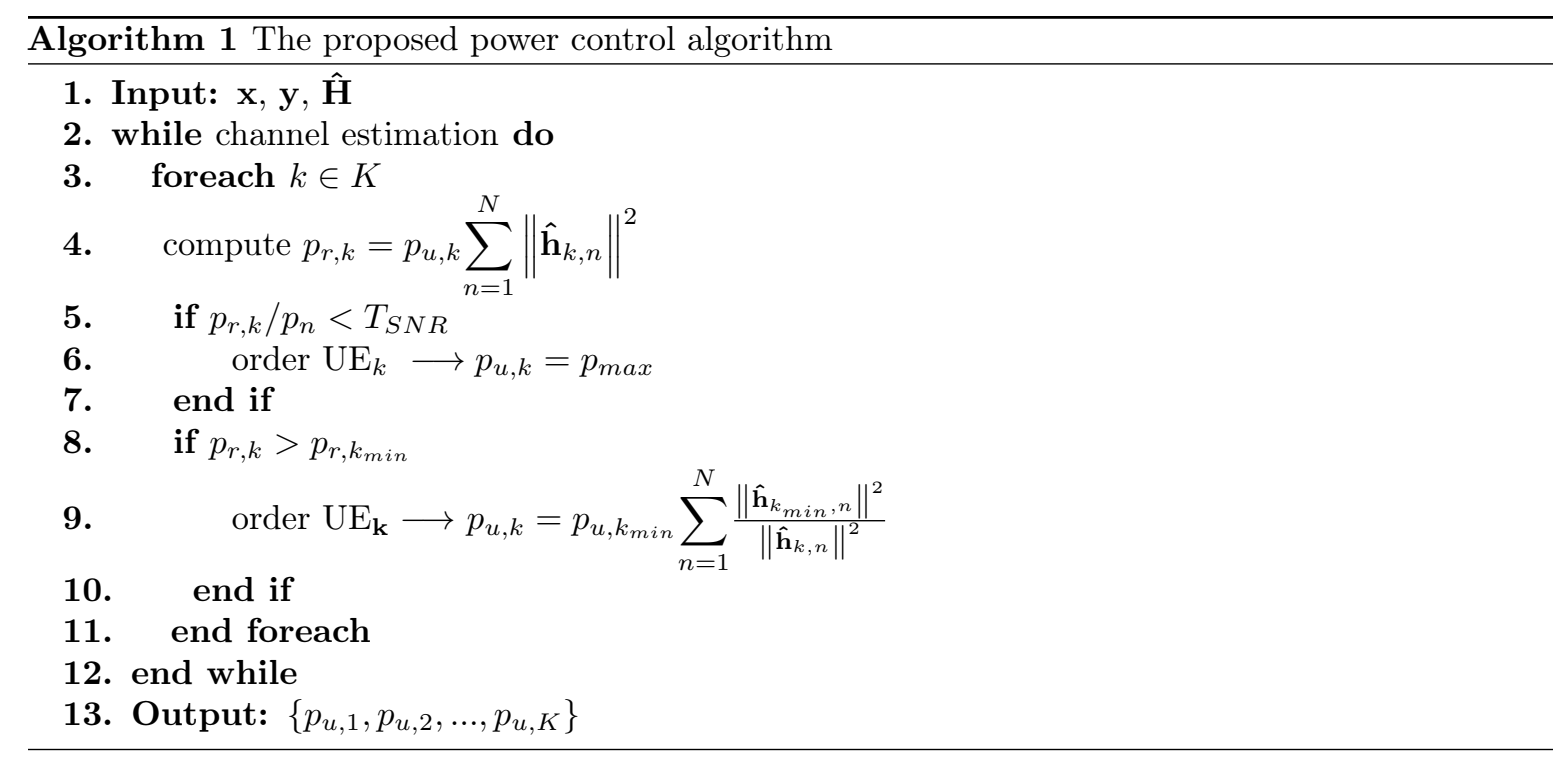

Table 2: Massive MIMO System Parameters

\begin{tabular}{|c|c|c|}
\hline Parameter & Description & Value \\
\hline \hline$M$ & Number of BS Antennas & 18 \\
\hline$K$ & Number of UEs & 8 \\
\hline$B$ & Total Bandwith & $500 \mathrm{MHz}$ \\
\hline$\Delta f$ & OFDM subcarrier bandwidth & $100 \mathrm{kHz}$ \\
\hline$N$ & Number of OFDM subcarriers & 5000 \\
\hline$p_{\max }$ & Maximum transmit power of UEs & $30 \mathrm{dBm}$ \\
\hline$R$ & Reflection attenuation coefficient & 0.5 \\
\hline$p_{n}$ & Noise power at $500 \mathrm{MHz}$ & $-80 \mathrm{dBm}$ \\
\hline \multicolumn{2}{|c}{ Modulation Scheme } & $128-\mathrm{QAM}$ \\
\hline
\end{tabular}

to operate during the CSI estimation as all UEs transmit predefined pilot symbols, hence, the BS can easily estimate the received powers and channel gains of all UEs at the same time. Then, during downlink, power control orders can be conveyed to the UEs. After power control, the average received power $\bar{p}_{r}$ from each UE will be similar as $\bar{p}_{r} \approx p_{r, k}$.

\section{$5 \quad$ Numerical Results}

This section presents OFDM massive MIMO system-level simulations using the set of channel data obtained by measurements. Although the channel measurements were performed with $700 \mathrm{MHz}$ bandwidth which is the $-3 \mathrm{~dB}$ bandwidth of the introduced antenna arrays, the system-level simulations are performed using the measured channel data with $500 \mathrm{MHz}$ bandwidth from $4.1 \mathrm{GHz}$ to $4.6 \mathrm{GHz}$ to ensure that the antenna gains are nearly constant by avoiding edges of the bandwidth. This bandwidth is divided into 5000 OFDM subcarriers resulting in $100 \mathrm{kHz}$ bandwidth for each. Table 2 presents the parameters that are used in the simulations. In addition to the measured channels, the favorable channel is obtained by (4). To obtain favorable channels, the Rician K-factor is selected as $\Lambda=0.1$ which provides rich scatterings by reducing the power of the LOS paths. The number of paths is $L=15$ and the UEs are randomly located between $2 \mathrm{~m}$ and $8 \mathrm{~m}$ distance to the BS as in the measurement scenario. The combined maximum antenna gain of the transmitter and the receiver antennas along each path is considered as $G_{m, k, n}=6 \mathrm{~dB}$ with $80^{\circ}$ on the E-plane and $100^{\circ}$ on the H-plane, the same as the radiation pattern of the introduced antenna.

The TDD mode includes pilot signaling, downlink, and uplink in a sequence. During pilot signaling, the UEs transmit orthogonal pilot symbols while the BS estimates the channel by the MMSE estimator. Firstly, channel estimation errors occurred using both arrays are examined. Fig. 13 illustrates minimum square error (MSE) of the channel estimation at different SNR values when the URA or SRA is employed at the BS. It clearly shows that the channel estimation errors are higher in the measured channels compared to the i.d.d. channel due to the channel correlation and received power imbalance which are observed between measured channels. The MMSE channel estimator performed better when the SRA was employed since it has lower mutual coupling and channel correlation compared to the URA as previously demonstrated.

After CSI estimation, the uplink throughput of the network is investigated using 128-QAM modulation. Fig. 14 illustrates the throughput of the network based on measured channel data when the URA or the SRA is employed and compares their performance to the i.d.d. channel as a function of SNR. It also shows the capacity bounds of the network. Differences between the channel capacities and the simulation results at lower SNR values are observed due to employing a high order modulation scheme. This figure shows that SRA outperforms URA in terms of throughput and a substantial capacity increase gained by employing power control in addition to decreasing power 


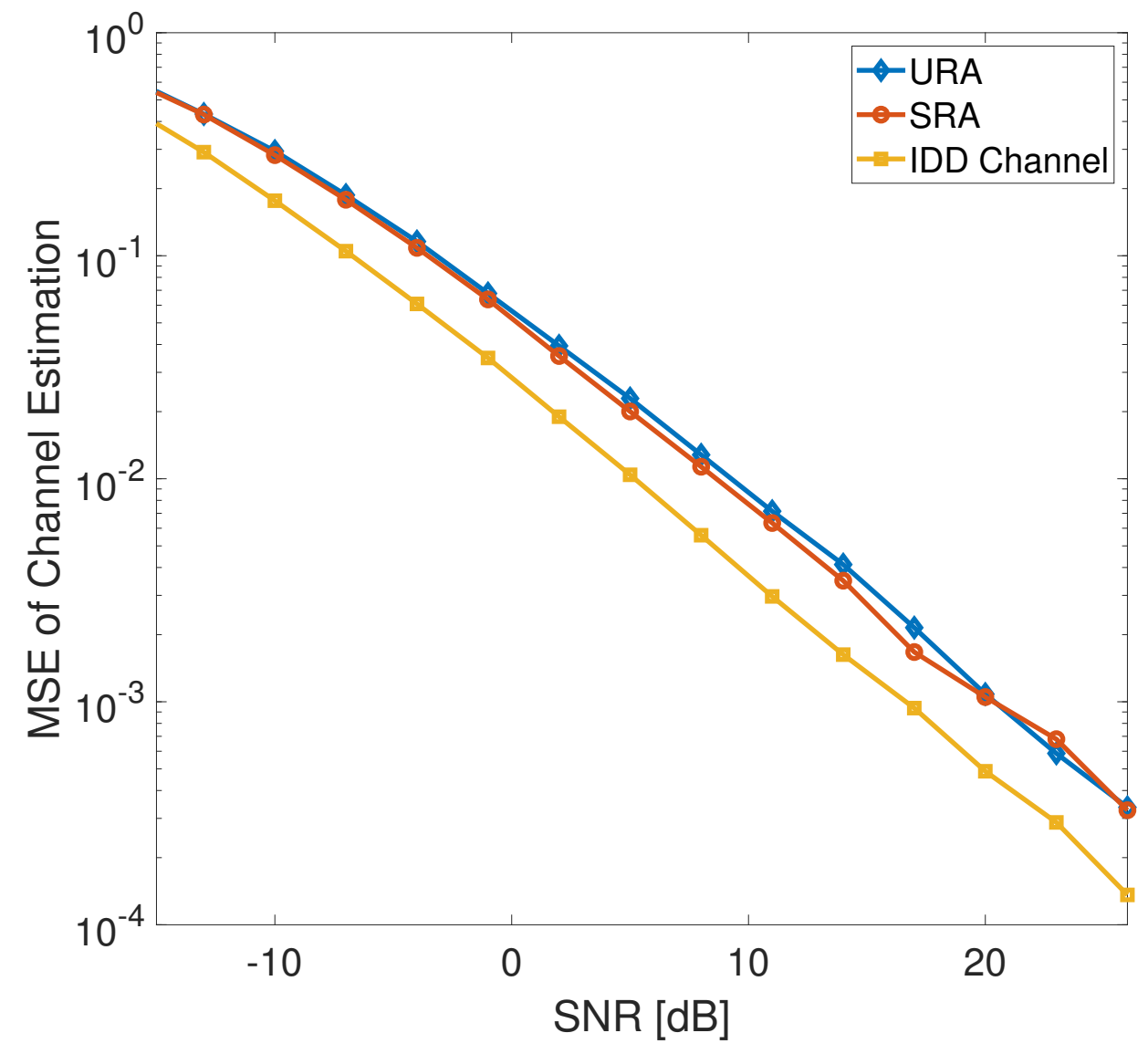

Fig. 13: CSI estimation errors with MMSE estimator. .

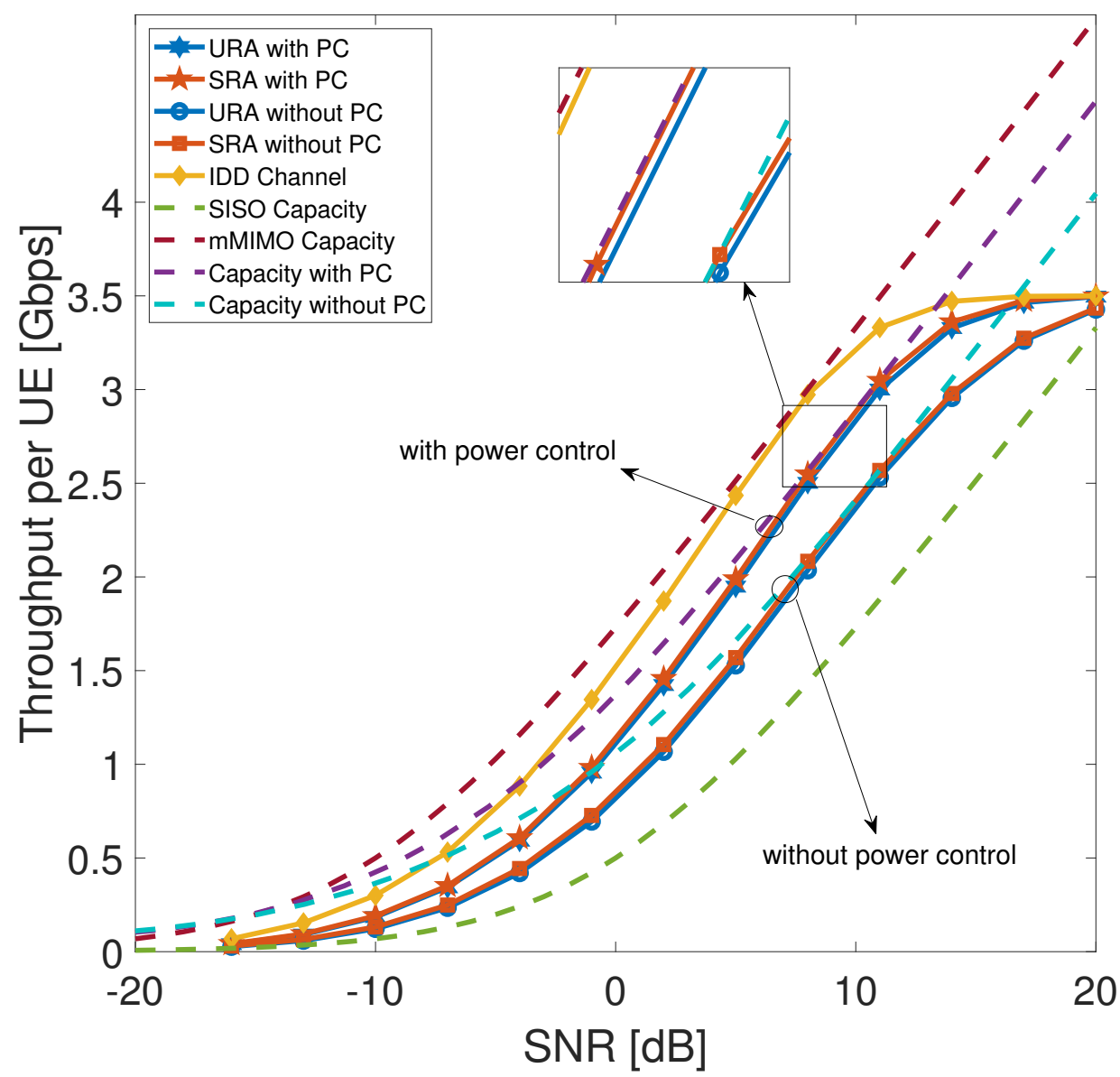

Fig. 14: Throughput per UE based on the measured channel data obtained with two arrays. The solid lines indicate the simulation results with 128-QAM-OFDM. capacities. 


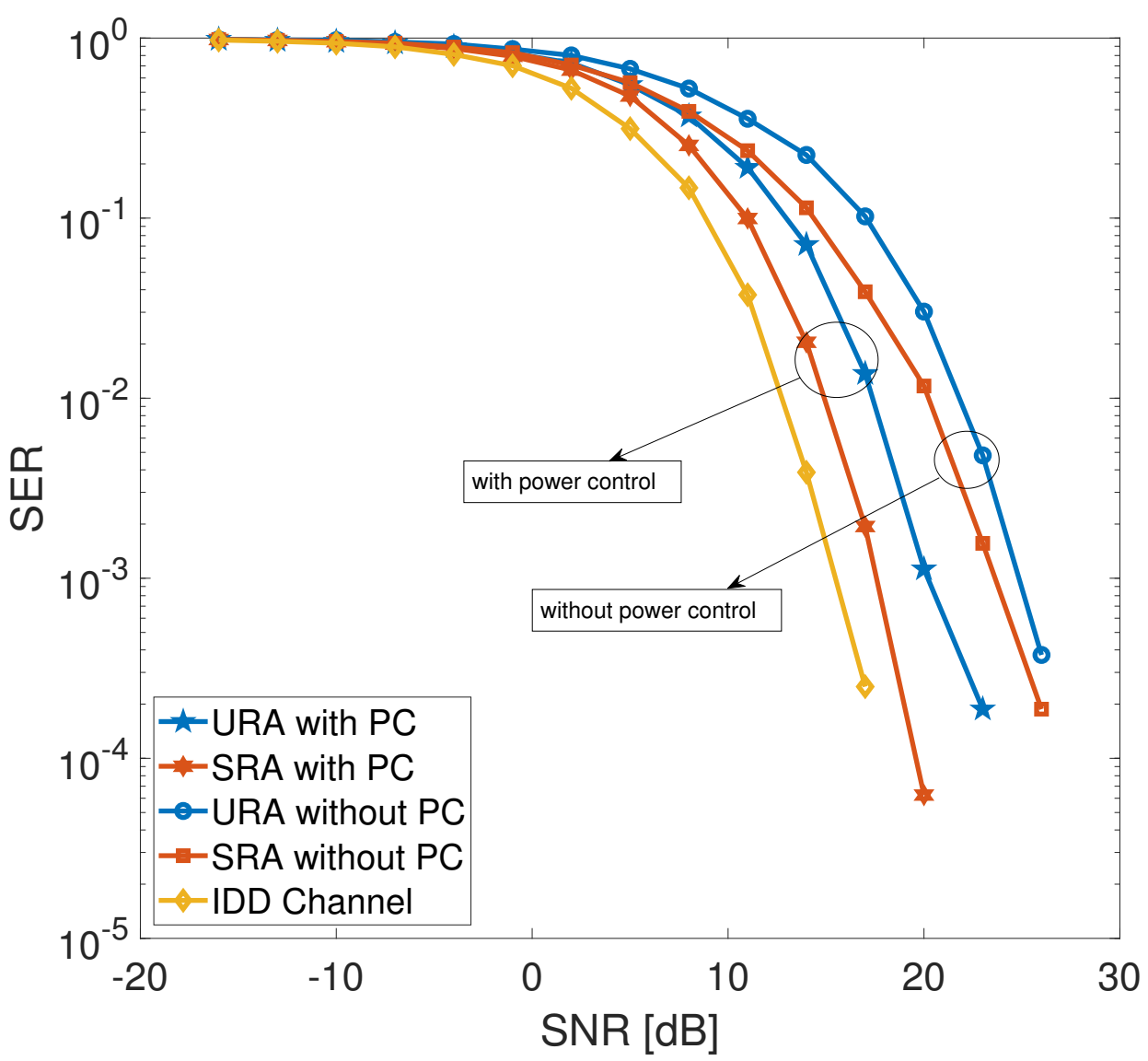

Fig. 15: SER with different arrays with and without PC.

consumption of the UEs. This confirms the importance of the PC algorithm for massive MIMO uplink to minimize interference and symbol errors. The average Symbol Error Rate (SER) is another important performance metric to evaluate the system performance, which is presented in Fig. 15 where a significant SER difference between these two antenna array geometries are observed.

These results show that the introduced array geometry has lower mutual coupling than the URA and alleviates the channel correlation in LOS, which leads to achieving a better capacity. Taking into consideration that these arrays occupy exactly the same physical size and cost the same, it would be beneficial to utilize the SRA geometry in indoor massive MIMO BS/APs. It is also shown that even with less than $0.5 \lambda$ element separation i.e. $0.36 \lambda$, which provides a decrease in the size of the array, massive MIMO networks can perform satisfactorily. Considering the physical size of the $3 \times 6$ arrays, they are sufficiently small to fit into a WIFI access point and can substantially improve the network capacity.

\section{Conclusion}

This study has introduced an antenna array geometry, namely SRA, for massive MIMO communication systems and comprehensively analysed its performance by experimental channel measurements. The proposed array geometry has been compared to the URA geometry in terms of mutual coupling, channel correlation, and network throughput. The results show that the SRA outperforms the URA in terms network capacity, by having a lower level of mutual coupling among the antenna elements, and less channel correlation, especially in LOS propagation. Moreover, a practical uplink power control algorithm has been proposed to enhance the network capacity further and its performance is investigated through the measured channel data. This study has demonstrated that even with a relatively small size antenna array massive MIMO can provide high throughput to multiple UEs at the same time under various channel conditions. Since the mobility of UEs is very low in indoor environments, implementing massive MIMO APs is a promising solution for high-density indoor networks such as in shared modern offices.

\section{References}

[1] M. Temiz, Y. Zhang, E. Alsusa, and L. Danoon, "Investigation of channel correlation in indoor wideband massive MIMO systems," in 2019 IEEE International Symposium on Antennas and Propagation and USNC-URSI Radio Science Meeting, pp. 1577-1578, July 2019.

[2] H. Q. Ngo, E. G. Larsson, and T. L. Marzetta, "Energy and spectral efficiency of very large multiuser MIMO systems," IEEE Transactions on Communications, vol. 61, pp. 1436-1449, April 2013. 
[3] À. O. Martínez, J. Ø. Nielsen, E. De Carvalho, and P. Popovski, "An experimental study of massive MIMO properties in 5G scenarios," IEEE Transactions on Antennas and Propagation, vol. 66, pp. 7206-7215, Dec 2018.

[4] X. Gao, O. Edfors, F. Rusek, and F. Tufvesson, "Massive MIMO performance evaluation based on measured propagation data," IEEE Transactions on Wireless Communications, vol. 14, pp. 3899 3911, July 2015.

[5] J. Chen, X. Yin, X. Cai, and S. Wang, "Measurement-based massive MIMO channel modeling for outdoor LoS and NLoS environments," IEEE Access, vol. 5, pp. 2126-2140, 2017.

[6] Q. Wang, B. Ai, D. W. Matolak, R. He, K. Guan, Z. Zhong, and D. Li, "Spatial variation analysis for measured indoor massive MIMO channels," IEEE Access, vol. 5, pp. 20828-20840, 2017.

[7] C. Masouros, M. Sellathurai, and T. Ratnarajah, "Large-scale MIMO transmitters in fixed physical spaces: The effect of transmit correlation and mutual coupling," IEEE Transactions on Communications, vol. 61, pp. 2794-2804, July 2013.

[8] C. T. Neil, A. Garcia-Rodriguez, P. J. Smith, P. A. Dmochowski, C. Masouros, and M. Shafi, "On the performance of spatially correlated large antenna arrays for millimeter-wave frequencies," IEEE Transactions on Antennas and Propagation, vol. 66, pp. 132-148, Jan 2018.

[9] X. Gao, O. Edfors, F. Tufvesson, and E. G. Larsson, "Massive MIMO in real propagation environments: Do all antennas contribute equally?," IEEE Transactions on Communications, vol. 63, pp. 3917-3928, Nov 2015.

[10] C. Chen, V. Volski, L. Van der Perre, G. A. E. Vandenbosch, and S. Pollin, "Finite large antenna arrays for massive MIMO: Characterization and system impact," IEEE Transactions on Antennas and Propagation, vol. 65, pp. 6712-6720, Dec 2017.

[11] X. Ge, R. Zi, H. Wang, J. Zhang, and M. Jo, "Multi-user massive MIMO communication systems based on irregular antenna arrays," IEEE Transactions on Wireless Communications, vol. 15, pp. 5287-5301, Aug 2016.

[12] T. Arai, A. Ohta, Y. Shirato, S. Kurosaki, K. Maruta, T. Iwakuni, and M. Iizuka, "Antenna array arrangement for massive MIMO to reduce channel spatial correlation in LOS environment," IEICE Transactions on Communications, vol. 100, no. 4, pp. 594-601, 2017.

[13] K. Maruta, T. Arai, A. Ohta, S. Kurosaki, T. Iwakuni, and M. Iizuka, "Experimental verification of massive antenna systems employing parallelogram planar arrays," IEEJ Transactions on Electrical and Electronic Engineering, vol. 12, pp. S82-S90, 2017.

[14] M. Gauger, J. Hoydis, C. Hoek, H. Schlesinger, A. Pascht, and S. t. Brink, "Channel measurements with different antenna array geometries for massive MIMO systems," in SCC 2015; 10th International ITG Conference on Systems, Communications and Coding, pp. 1-6, Feb 2015.

[15] M. Bennis, M. Simsek, A. Czylwik, W. Saad, S. Valentin, and M. Debbah, "When cellular meets WiFi in wireless small cell networks," IEEE Communications Magazine, vol. 51, no. 6, pp. 44-50, 2013.

[16] Zhi Ning Chen and M. Y. W. Chia, "A novel center-slot-fed suspended plate antenna," IEEE Transactions on Antennas and Propagation, vol. 51, no. 6, pp. 1407-1410, 2003.

[17] X. N. Low, Z. N. Chen, and W. K. Toh, "Ultrawideband suspended plate antenna with enhanced impedance and radiation performance," IEEE Transactions on Antennas and Propagation, vol. 56, pp. 2490-2495, Aug 2008.

[18] "Precompliance EMC test antenna HyperLOG 30xxx series logarithmic periodic antenna - 380MHz to $18 \mathrm{GHz}$, Aaronia AG," Sept 2014.

[19] H. Wei, D. Wang, H. Zhu, J. Wang, S. Sun, and X. You, "Mutual coupling calibration for multiuser massive MIMO systems," IEEE Transactions on Wireless Communications, vol. 15, pp. 606-619, Jan 2016.

[20] X. Chen, S. Zhang, and Q. Li, "A review of mutual coupling in MIMO systems," IEEE Access, vol. 6 , pp. 24706-24719, 2018

[21] M. Temiz, E. Alsusa, and L. Danoon, "Impact of imperfect channel estimation and antenna correlation on quantised massive multiple-input multiple-output systems," IET Communications, vol. 13, no. 9, pp. 1262-1270, 2019.

[22] E. Björnson, J. Hoydis, and L. Sanguinetti, Massive MIMO Networks: Spectral, Energy, and Hardware Efficiency. Now Publishers, 2017.

[23] R. Zhang, M. Wang, L. X. Cai, Z. Zheng, X. Shen, and L. Xie, "LTE-unlicensed: the future of spectrum aggregation for cellular networks," IEEE Wireless Communications, vol. 22, pp. 150-159, June 2015. 
[24] D. Rosolowski, D. Gryglewski, P. Korpas, W. Wojtasiak, and J. Modelski, "An ultrawideband 1 to $6 \mathrm{GHz}$ 0-IF radio receiver with $500 \mathrm{MHz}$ of instantaneous bandwidth," in 2016 21st International Conference on Microwave, Radar and Wireless Communications (MIKON), pp. 1-4, May 2016.

[25] J. Medbo, P. Kyosti, K. Kusume, L. Raschkowski, K. Haneda, T. Jamsa, V. Nurmela, A. Roivainen, and J. Meinila, "Radio propagation modeling for $5 \mathrm{G}$ mobile and wireless communications," IEEE Communications Magazine, vol. 54, pp. 144-151, June 2016.

[26] E. Torabi and A. Ghorbani, "Reflection coefficient modification for mobile path loss calculation," in Proceedings of the International Conference Days on Diffraction-2005, vol. 0, pp. 94-108, June 2005.

[27] H. Yin, D. Gesbert, M. Filippou, and Y. Liu, "A coordinated approach to channel estimation in large-scale multiple-antenna systems," IEEE Journal on Selected Areas in Communications, vol. 31, pp. 264-273, February 2013.

[28] P. He, L. Zhao, S. Zhou, and Z. Niu, "Water-filling: A geometric approach and its application to solve generalized radio resource allocation problems," IEEE Transactions on Wireless Communications, vol. 12, pp. 3637-3647, July 2013. 